\title{
Long-run heterogeneity in an exchange economy with fixed-mix traders
}

\section{Giulio Bottazzi, Pietro Dindo \& Daniele Giachini}

\section{Economic Theory}

ISSN 0938-2259

Econ Theory

DOI 10.1007/s00199-017-1066-8



照 Springer 
Your article is protected by copyright and all rights are held exclusively by SpringerVerlag GmbH Germany. This e-offprint is for personal use only and shall not be selfarchived in electronic repositories. If you wish to self-archive your article, please use the accepted manuscript version for posting on your own website. You may further deposit the accepted manuscript version in any repository, provided it is only made publicly available 12 months after official publication or later and provided acknowledgement is given to the original source of publication and a link is inserted to the published article on Springer's website. The link must be accompanied by the following text: "The final publication is available at link.springer.com". 


\title{
Long-run heterogeneity in an exchange economy with fixed-mix traders
}

\author{
Giulio Bottazzi ${ }^{2} \cdot$ Pietro Dindo $^{1,2}$ (D) Daniele Giachini ${ }^{2}$
}

Received: 15 June 2016 / Accepted: 12 July 2017

(C) Springer-Verlag GmbH Germany 2017

\begin{abstract}
We consider an exchange economy where agents have heterogeneous beliefs and assets are long-lived, and investigate the coupled dynamics of asset prices and agents' wealth. We assume that agents hold fixed-mix portfolios and invest on each asset proportionally to its expected dividends. We prove the existence and uniqueness of a sequence of arbitrage-free market equilibrium prices and provide sufficient conditions for an agent, or a group of agents, to survive or dominate. Our main finding is that long-run coexistence of agents with heterogeneous beliefs, leading to asset prices endogenous fluctuations, is a generic outcome of the market selection process.
\end{abstract}

Keywords Market selection hypothesis - Heterogeneous beliefs · Asset pricing · Evolutionary finance $\cdot$ Incomplete markets

JEL Classification C60 - D52 - D53 - G11 - G12

\begin{abstract}
We wish to thank the editor, the associate editor, and two anonymous reviewers for their helpful comments and suggestions. Pietro Dindo gratefully acknowledges the support of the Marie Curie International Outgoing Fellowship PIOF-GA-2011-300637 within the 7th European Community Framework Programme and the hospitality of the Department of Economics at Cornell University. Daniele Giachini gratefully acknowledges the hospitality of the Department of Banking and Finance at the University of Zurich.
\end{abstract}

$凶 \quad$ Pietro Dindo

pietro.dindo@unive.it

1 Dipartimento di Economia, Università Ca’ Foscari Venezia, San Giobbe, Cannaregio 873, 30121 Venezia, Italy

2 Istituto di Economia, Scuola Superiore Sant'Anna, Piazza Martiri della Libertà 33, 56127 Pisa, Italy 


\section{Introduction}

The market selection hypothesis (MSH) applied to financial markets implies that traders' heterogeneity can be only a short-run phenomenon. In the long run, the trader with the most accurate beliefs about asset dividends should gain all the wealth and price assets accordingly. Indeed, benchmark equilibrium models of asset pricing, such as Lucas' model and the CAPM, dismiss heterogeneity and assume that all traders have correct beliefs about the distribution of asset returns. Although these models provide an insightful characterization of the relation between equilibrium returns and risk preferences, they have not been validated by data. ${ }^{1}$

In this paper, we investigate whether, contrary to what the MSH would suggest, traders' heterogeneity can persist in financial markets. We study a discrete-time Lucas' tree economy, as in Lucas (1978), but assume that agents are heterogeneous and invest in assets using fixed-mix portfolios. In particular, we specify subjective beliefs for each agent and assume that they invest on each asset proportionally to its expected dividends. We ask whether the market selects for a unique agent or, instead, multiple agents survive and determine equilibrium prices in the long run. The contribution of our paper is twofold. Firstly, we show that even in a setting where portfolio rules are not explicitly derived from an intertemporal utility maximization, there exists a unique sequence of arbitrage-free market equilibrium prices. Secondly, we provide sufficient conditions for the dominance, vanishing, or survival of groups of agents. These conditions depend on the relative performance of portfolios in the limit of a group having all the wealth of the economy. In relation to the original question, we finally use these conditions to show that long-run heterogeneity is a generic outcome of the market selection process.

The formal investigation of the MSH has started only many years after its formulation by Alchian (1950) and Friedman (1953). The seminal work by Blume and Easley (1992) has led to two strands of literature. In the first group, agents are expected utility maximizers, have rational price expectations, but disagree on the dividend process, see, e.g., Sandroni (2000), Blume and Easley (2006), and Jouini and Napp (2006) for discrete-time models and Jouini and Napp (2007), Yan (2008), Cvitanić et al. (2012), and Bhamra and Uppal (2014) for continuous-time models. The main finding is that when markets are complete they do select for a unique trader. Heterogeneity is only transient and assets are priced by the surviving agent. ${ }^{2,3}$

\footnotetext{
${ }^{1}$ For a list of puzzles and asset pricing anomalies, see, e.g., the entries "Financial Market Anomalies" and "Finance (new developments)" in the New Palgrave Dictionary of Economics.

2 See however, Cvitanić and Malamud (2011) for a distinction between the price and portfolio impact of a vanishing agent and Cvitanić et al. (2012) for an appraisal of the impact of vanishing agents on cumulated returns.

${ }^{3}$ Heterogeneity may instead be persistent when markets are exogenously incomplete (Blume and Easley 2006; Beker and Chattopadhyay 2010; Coury and Sciubba 2012), when agents have non-tradable labor income (Cao 2013; Cogley et al. 2013), when information is asymmetric and costly (Sciubba 2005), when learning does not converge (Sandroni 2005; Beker and Espino 2011), when agents are ambiguity averse (Condie 2008; Guerdjikova and Sciubba 2015), or when agents have recursive preferences (Borovička 2015; Dindo 2015).
} 
Another strand of literature, to which this work belongs, has instead investigated the market selection process in economies where agents' behavior can be modeled directly in terms of saving and portfolio rules, not necessarily coming from expected utility maximization under rational price expectations (see, e.g., Sciubba 2006; Evstigneev et al. 2009, and the chapters in Hens and Schenk-Hoppé (2009)). These works contend that agents are able to coordinate on having rational expectations on future prices, especially when they disagree on the dividend process, and prefer to assume that agents' investment strategies are given adapted processes. ${ }^{4}$ The question is whether also in this more realistic setup the market selects for a unique portfolio. ${ }^{5}$

An interesting result of this second strand of literature is that, provided saving is homogeneous, there exists a portfolio rule that dominates against any other combination of adapted rules, as shown in Evstigneev et al. (2002, 2006, 2008). This portfolio, named Generalized Kelly after Kelly (1956), invests on each asset proportionally to its expected dividends with expectations computed using the correct dividend process probability. In particular, Evstigneev et al. (2008) establish the global dominance of the Generalized Kelly rule in an i.i.d. exchange economy where agents can trade multiple long-lived assets. Given the log-optimality of the Generalized Kelly portfolio when the agent using it is alone in the economy, the predictions of Lucas' model (with a log-utility maximizer) are recovered in the limit and the MSH is validated. However, in markets where all agents use a fixed-mix rule the result holds only if there is an agent who (i) invests on each asset proportionally to its expected dividends and (ii) in every period knows the distribution of next period's dividends. The latter is a very strong assumption in real financial markets; hence, a relevant question remains: Is the market able to select for the most accurate beliefs when nobody is always right? ${ }^{6}$ Otherwise, is coexistence of heterogeneous fixed-mix rules a possible outcome of market selection?

In order to provide an answer to these questions, we proceed as follows. In Sect. 2, we introduce an asset market economy where agents hold heterogeneous fixed-mix portfolios of long-lived assets. In particular, given subjective beliefs, we assume that agents invest on assets proportionally to their expected dividends. We name these portfolios Subjective Generalized Kelly. In Sect. 3, we determine the dynamics of agents' wealth and asset prices starting from budget constraints and market clearing equations. Although the two dynamics are coupled-since assets are long-lived their payoffs determine the new wealth distribution but the new wealth distribution determines, through prices, asset payoffs-we solve them explicitly and provide an expression of asset payoffs that depends only on agents' rules and on their old wealth distribution. We use this characterization of asset payoffs to explicit conditions that exclude arbitrage. Subjective Generalized Kelly portfolios naturally satisfy these conditions. We can thus show that in our markets there exists a unique sequence of no-arbitrage market

\footnotetext{
4 In particular, why should (endogenously determined) prices be easier to forecast than (exogenously given) dividends?

5 The empirical evidence is that it does not, there were 9520 mutual funds in the USA in 2015.

6 Bottazzi and Dindo (2014) investigate the same issue in an economy with short-lived assets, finding that the MSH does not generally hold. Bektur (2013) shows that the agent whose rule is the closest, component by component, to the Generalized Kelly rule survives almost surely.
} 
equilibrium prices. Although previous works, namely Evstigneev et al. (2006, 2008), also provide conditions for the existence and uniqueness of a sequence of positive prices in a model very close to ours, the result on arbitrages is new.

In Sect. 4, we proceed to provide sufficient conditions for a group of agents to have a positive, null, or unitary, fraction of wealth in the long run. These conditions are based on the comparison of groups' relative growth rates, themselves a function of aggregate portfolios, in the limit when a group has all the assets, and thus all the wealth of the economy. Given a partition $\{I, J\}$ of the entire set of agents, it is intuitive that if, for all market conditions set by $J$, group $I$ has "better" portfolios than group $J$, in that it has a higher average log growth rate, then $I$ cannot vanish. These survival results lead also to dominance provided (i) group $I$ is also performing, on average, better than group $J$ at the market conditions set by $I$ and (ii) no arbitrages are possible so that the groups' wealth ratios keep changing. In the simplest case of a two-agent economy, analyzed in Sect. 4.1, these sufficient conditions are also necessary (but for hairline cases) and particularly easy to check. The reason is that when an agent dominates, assets payoffs become unequivocally defined.

Using our sufficient conditions for survival, we are able to characterize when long-run heterogeneity occurs. Our main finding is that agents with heterogeneous portfolios, and thus heterogeneous beliefs, may have positive wealth in the long run. In this case, the relative wealth distribution changes over time, so that different portfolios have a different impact on asset prices in different periods. The price distribution depends on the distribution of relative wealth and has as support the subset of the simplex defined by agents' beliefs. In Sect. 4.2, we show that long-run heterogeneity occurs in all economies, that is, no matter the exact asset structure and the number of agents, and is generic, that is, it does not disappear if agents' beliefs are locally perturbed. The survival of different agents is related to portfolios and dividends being anti-correlated: If an agent invests more in the asset that pays more in one state while the other agent invests more in an asset that pays more in another state, then the outcome is long-run heterogeneity. ${ }^{7}$

The difference between our results and those of the general equilibrium literature with dynamically complete markets of long-lived assets, as Sandroni (2000) or Yan (2008), lies in the non-optimality of Subjective Generalized Kelly rules. Consider an economy with two agents, $i$ and $j$, and assume that the beliefs of $i$ are more accurate. When both agents hold log-optimal portfolios, agent $i$ dominates and agent $j$ vanishes. If, instead, we find that $j$ does not vanish, then it must be that agent $j$ 's non-optimal portfolio is "better" than the optimal portfolio derived under her beliefs, at least in the limit when agent $i$ has most of the wealth. Thus, the non-optimality of agent $j$ 's portfolio corrects for the inaccuracy of her beliefs, leading to "better" portfolios and to her survival. ${ }^{8}$

\footnotetext{
7 The result seems related to the analysis of the impact of pessimism and optimism on asset prices performed in Jouini and Napp (2010). Note, however, that their result is non-generic in that it holds only when agents' biases are equal, so that they have the same survival index. See also Blume and Easley (2009). Our results are instead generic.

8 Note, however, that survival might not be associated with higher welfare (see, e.g., Jouini and Napp 2016).
} 
We provide an intuition of the interplay between non-correct beliefs and the nonoptimality of portfolios, by defining an agent effective beliefs as those (time-varying) beliefs such that the Subjective Generalized Kelly rule derived using the original beliefs coincides with the log-optimal portfolio rule derived using effective beliefs (and rational price expectations). Since a Subjective Generalized Kelly portfolio is log-optimal when the agent using it has all the wealth, effective beliefs and beliefs coincide in this case. However, when asset returns are determined by both agents, they differ. In a two-agent economy, long-run heterogeneity occurs if agent $i$ 's effective beliefs are more accurate than agent $j$ 's beliefs when asset returns are determined by agent $j$ (because she has most of the wealth) and, at the same time, agent $j$ 's effective beliefs are more accurate than agent $i$ 's beliefs when asset returns are determined by agent $i$. In these cases, there exists a compensation between using a non-optimal portfolio rule and having non-accurate beliefs.

We discuss our results in terms of effective beliefs in Sect. 5. Here we also show, by means of numerical explorations, that long-run heterogeneity occurs for a wide range of the economy parameters. Moreover, although throughout the paper we concentrate on the selection of Subjective Generalized Kelly portfolios, in Sect. 5.4 we provide an example where fixed-mix portfolios are derived to be optimal when the agent using it has all the wealth of the economy and different risk preferences then a log-utility maximizer. We show that even if all agents know the truth, long-run heterogeneity still occurs generically. Section 6 concludes.

\section{The model}

Time is discrete and indexed by $t \in \mathbb{N}_{0}=\mathbb{N} \cup\{0\}$. At each date $t \in \mathbb{N}$ one of the possible $S=\{1, \ldots, S\}$ states of the world occurs. Let $\Sigma$ be the set of all possible sequences of states of the world. We denote with $\sigma$ a generic sequence in $\Sigma$, with $s_{t} \in S$ the state of the world realized at date $t$, and with $\sigma_{t}=\left(s_{1}, s_{2}, \ldots, s_{t}\right) \in S^{t}$ the partial history of states up to date $t$ included. $\left\{\mathfrak{I}_{t}\right\}$ is the natural filtration, $\mathfrak{\Im}$ is the $\sigma$-algebra generated by the filtration, and $\mathrm{P}$ is a probability measure on $(\Sigma, \Im)$. All the random variables on $(\Sigma, \Im)$ that we shall introduce (dividends, asset prices, portfolios, wealth, etc.) are adapted to the natural filtration $\left\{\mathfrak{\Im}_{t}\right\}$. Unless otherwise noted, all our statements are true almost surely with respect to the probability measure $\mathrm{P}$.

We consider an exchange economy populated by $N$ agents trading $K$ long-lived assets. Asset $k \in K$ traded in $t \in \mathbb{N}_{0}$ pays the dividend $D_{k, t^{\prime}}\left(\sigma_{t^{\prime}}\right)$ in $t^{\prime}>t$. Agents have no other source of income, so that the aggregate endowment $Y_{t}$ is the sum of the payed dividends, $Y_{t}\left(\sigma_{t}\right)=\sum_{k \in K} D_{k, t}\left(\sigma_{t}\right)$. Denote asset prices at date $t$ as $P_{t}=$ $\left(P_{1, t}, \ldots, P_{K, t}\right)$, the asset holding of agent $i$ at date $t$ as $h_{t}^{i}=\left(h_{1, t}^{i}, \ldots, h_{K, t}^{i}\right)$, and her consumption in $t$ as $C_{t}^{i}$. Agent $i$ 's budget constraint in $t \geq 1$ reads

$$
C_{t}^{i}+\sum_{k=1}^{K} P_{k, t} h_{k, t}^{i}=\sum_{k=1}^{K}\left(D_{k, t}+P_{k, t}\right) h_{k, t-1}^{i}
$$

The budget constraint in $t=0$ is similar, but the right-hand side of (1) is the value of agent $i$ 's initial endowment of consumption good and assets. Asset prices are fixed 
in competitive markets. Without loss of generality, we assume unitary supply, so that the date- $t$ asset- $k$ market clearing condition reads

$$
\sum_{i=1}^{N} h_{k, t}^{i}=1
$$

The existence and uniqueness of positive market clearing prices depends on agents' demands. We postpone to Sect. 3 the proof that under appropriate assumptions on demands there exists a unique vector of arbitrage-free prices such that (1) and (2) hold for every $i \in N, k \in K$, and $t \in \mathbb{N}$.

We define agent $i$ 's wealth in $t \geq 1$ as her pre-consumption net worth so that

$$
W_{t}^{i}=\sum_{k=1}^{K}\left(D_{k, t}+P_{k, t}\right) h_{k, t-1}^{i}, \quad \forall i \in N
$$

and let $W_{t}=\left(W_{t}^{1}, \ldots, W_{t}^{N}\right)$ denote the vector of agents' wealth. Equations (1-2) can be rewritten in terms of agents' wealth. To this end, it is convenient to express agent $i$ 's consumption and investment decision in $t$ as fractions of her wealth $W_{t}^{i}$. We denote with $1-\delta_{t}^{i} \in(0,1)$ the fraction of wealth she consumes, so that $C_{t}^{i}=\left(1-\delta_{t}^{i}\right) W_{t}^{i}$, and with $\alpha_{t}^{i}=\left(\alpha_{1, t}^{i}, \ldots, \alpha_{K, t}^{i}\right)$ the vector of her investment fractions, so that $h_{k, t}^{i}=\alpha_{k, t}^{i} W_{t}^{i} / P_{k, t}$. By (1) and (3) $\sum_{k=1}^{K} \alpha_{k, t}^{i}=\delta_{t}^{i}$, i.e., $\delta_{t}^{i}$ is agent- $i$-date- $t$ saving rate. Using agents' wealth and investment decisions, Eqs. (1-2) in $t \geq 1$ become, respectively,

$$
\begin{aligned}
W_{t}^{i} & =\sum_{k=1}^{K}\left(D_{k, t}+P_{k, t}\right) \frac{\alpha_{k, t-1}^{i} W_{t-1}^{i}}{P_{k, t-1}}, \quad \forall i \in N, \\
P_{k, t} & =\sum_{i=1}^{N} \alpha_{k, t}^{i} W_{t}^{i}, \quad \forall k \in K .
\end{aligned}
$$

Since assets are long-lived, the dynamics of agents' wealth and asset prices are coupled. Before solving (4-5), we specify asset dividends and agents' consumption-investment decisions.

\subsection{Assets}

The following assumptions specify the nature of asset dividends. First, we shall assume that each asset $k$ 's relative dividend process, $D_{k, t} / Y_{t}$, does not depend on partial histories. Second, we shall restrict the probability measure $\mathrm{P}$ so that, in all periods, relative dividends have the same distribution. In other words, there exist a strictly positive probability measure $\pi$ on $\left(S, 2^{S}\right)$ and $K$ random variables on $\left(S, 2^{S}\right),\left(d_{1}, \ldots, d_{K}\right)=d$, such that

D1 $D_{k, t}\left(\sigma_{t}\right)=d_{k}\left(s_{t}\right) Y_{t}\left(\sigma_{t}\right), \forall k \in K, \forall t \in \mathbb{N}$, and $\forall \sigma_{t} \in S^{t}$. 
D2 States of the world are independent and identically distributed with $\mathrm{P}\left(s_{t}=s\right)=$ $\pi(s)>0 \forall t \in \mathbb{N}, \forall \sigma \in \Sigma$, and $\forall s \in S$.

Throughout the paper, we shall identify distributions $\pi$ on $\left(S, 2^{S}\right)$ with vectors $\pi \in \Delta^{S}$ by using $\pi(s)=\pi_{s} \forall s \in S .{ }^{9}$ Given that the aggregate endowment is the sum of asset dividends, it follows that $\sum_{k=1}^{K} d_{k}(s)=1$ for every $s \in S$. Note that although D2 is a restriction on the relative dividend process, the aggregate endowment $Y_{t}$ can be a more general adapted process on $(\Sigma, \mathfrak{I}, \mathrm{P})$. Under D1 and D2

$$
\mathrm{E}^{\mathrm{P}}\left[D_{k, t} \mid \Im_{t-1}\right]=\mathrm{E}^{\pi}\left[d_{k}\right] \mathrm{E}^{\mathrm{P}}\left[Y_{t} \mid \Im_{t-1}\right], \quad \forall k \in K \text { and } \forall t \in \mathbb{N},
$$

where $\mathrm{E}$ is the expectation operator that integrates according to the measure indicated by the superscript. We also assume that dividends are nonnegative and that every asset pays a positive dividend in at least some states

D3 $d_{k}(s) \geq 0 \forall s \in S$ and $\mathrm{E}^{\pi}\left[d_{k}\right]>0, \forall k \in K$.

Finally, we rule out the existence of redundant assets. Defining the matrix $D$ with elements $d_{k, s}=d_{k}(s)$ for all $k \in K$ and $s \in S$, we assume

D4 $\operatorname{Rank}(D)=K \leq S$.

As we shall show, the dividend matrix $D$, rather than the aggregate process $\left\{Y_{t}\right\}$, is central to the analysis of agents' relative wealth dynamics. Some examples of dividend matrices follow.

Diagonal dividends Assume that there are as many assets as states, $K=S$, and that the dividend of asset $k$ in $t$ is the entire aggregate endowment if and only if state $s_{t}=k$ is realized. Using II to denote the identity matrix, with appropriate dimension, asset $k$ traded in $t$ pays the dividend

$$
D_{k, t^{\prime}}=\mathbb{I}_{k, s_{t^{\prime}}} Y_{t^{\prime}} \quad \text { in } \quad t^{\prime}>t
$$

Asset $k$ traded in $t$ is a bet on the occurrence of state $s_{t^{\prime}}=k$ for all $t^{\prime}>t$. By construction asset dividends are anti-correlated. The dividend matrix $D$ is just the $S \times S$ identity matrix and D1, D3, D4 are satisfied.

Binomial tree Here we construct the matrix $D$ that replicates the simplest canonical model of financial markets. Assume that the aggregate endowment follows a geometric random walk:

$$
Y_{t}= \begin{cases}g_{u} Y_{t-1} & \text { if } s_{t}=1 \\ g_{d} Y_{t-1} & \text { if } s_{t}=2\end{cases}
$$

\footnotetext{
${ }^{9}$ Given $\mathbb{R}^{S}, \Delta^{S}$ denotes its simplex, $\mathbb{R}_{+}^{S}$ is the subset of vectors with nonnegative components (excluding the null vector), and $\mathbb{R}_{++}^{S}$ is the subset of vectors with positive components.
} 
with $g_{u}>g_{d}$. Two assets in unitary supply are available. The first, $k=1$, is risky. When purchased in $t$, it has dividends in all $t^{\prime}>t$ equal to

$$
D_{1, t^{\prime}}=\left\{\begin{array}{cc}
\left(g_{u}-g_{d}\right) Y_{t^{\prime}-1} & \text { if } s_{t}=1 \\
0 & \text { if } s_{t}=2
\end{array} .\right.
$$

The second asset is risk-free. In all $t^{\prime}>t$, it has dividend $g_{d} Y_{t^{\prime}-1}$, independently of the state $s_{t^{\prime}}$. The asset is a perpetual bond with time-varying coupon. Since the first asset is equivalent to a long position in the aggregate endowment and a short position in the second asset, the market is equivalent to a market with a risk-free asset in zero supply and a risky asset, that pays the aggregate endowment as dividends, in unitary supply. The dividend matrix $D$ is found by imposing D1

$$
D=\left[\begin{array}{cc}
1-r & 0 \\
r & 1
\end{array}\right] \text {, }
$$

with $r=g_{d} / g_{u} \in(0,1)$. It can be easily checked that D3-D4 are satisfied too.

Trinomial tree In both previous examples, market completeness relies on the properties of the full payoff matrix, that is, on the sum of dividends and prices. Thus, even if $D$ is non-singular, the market might still be incomplete. However, when there are fewer assets than states, $K<S$, we know for sure that asset markets are incomplete. A strength of our approach is that we are able to analyze long-run outcomes of the economy also for these incomplete markets.

Consider for example an economy with $S=3$ and three possible aggregate endowment growth rates: $g_{u} \geq g_{m}>g_{d}$. Only two assets are traded. As in the previous example, the first contract is a long position in the aggregate endowment and a short position in the risk-free asset paying the dividend $g_{d} Y_{t^{\prime}-1}$ in all $t^{\prime}>t$. The dividend matrix $D$ is

$$
D=\left[\begin{array}{ccc}
1-r_{u} & 1-r_{m} & 0 \\
r_{u} & r_{m} & 1
\end{array}\right],
$$

where $r_{u}=g_{d} / g_{u} \leq r_{m}=g_{d} / g_{m}$ (also in this case also D3-D4 are satisfied). Assume now that the first contract is replaced by two contracts that can disentangle the position in the first and second state of the economy. Simple computations show that the dividend matrix is non-singular and given by

$$
D=\left[\begin{array}{ccc}
1-r_{u} & 0 & 0 \\
0 & 1-r_{m} & 0 \\
r_{u} & r_{m} & 1
\end{array}\right]
$$

\subsection{Investment rules}

Although one could study the market dynamics with general investment rules $\alpha \mathrm{s}$, throughout this work we concentrate on a special class of rules, fixed-mix rules. We 
assume that each agent $i$ invests a constant and positive fraction $\alpha_{k}^{i}$ on each asset $k$. In particular, each agent $i$ has a constant saving rate $\delta^{i}$ and chooses $\alpha^{i}$ such that her portfolio $x^{i}=\alpha^{i} / \delta^{i}$ is the Generalized Kelly portfolio of Evstigneev et al. (2002, 2006, 2008) computed under her subjective beliefs.

R1 Each agent $i \in N$ has constant saving rate $\delta^{i} \in(0,1)$, constant subjective beliefs $\pi^{i}$ on $\left(S, 2^{S}\right)$, and for all $t \in \mathbb{N}_{0}$ uses the fixed-mix investment rule $\alpha^{i}=\delta^{i} x^{i}$ with portfolio $x^{i}=\mathrm{E}^{\pi^{i}}[d]$.

We further assume that each agent believes that all states are possible: ${ }^{10}$

R2 $\pi^{i}$ is strictly positive $\forall i \in N$.

A portfolio rule that satisfies $\mathbf{R 1 - R 2}$ is named Subjective Generalized Kelly rule. Given R1-D1, investment shares are constant, i.e., fixed. Given R2-D3 its investment shares are sufficiently mixed, $\alpha_{k}^{i}>0$ for all $k \in K$ and all $i \in N$. As a result, a Subjective Generalized Kelly is indeed fixed-mix in our market economy. Note that rules can also allow some form of short selling, as long as the aggregate position in the existing assets is positive (see the examples in the previous section). It is particularly important to realize that, given restrictions R1-R2, the set of consumption allocations that agent $i$ can purchase and the long-run dynamics of the model depend critically on $D$. Given two different dividend matrices $D$ and $D^{\prime}$, and two sequences of prices $P$ and $P^{\prime}$ such that the law of one price holds, there might not exist a pair of portfolio rules $x$ and $x^{\prime}$ satisfying $\mathbf{R} 1-\mathbf{R} 2$ such that the stream of payoffs is the same for $x$ under $D$ and $P$ and for $x^{\prime}$ under $D^{\prime}$ and $P^{\prime}$.

We focus our analysis on Subjective Generalized Kelly rules because they represent a benchmark in the space of fixed-mix rules. Specifically, when subjective beliefs are correct we have the Generalized Kelly rule of Evstigneev et al. (2008). In an i.i.d. economy, such rule gains all the aggregate endowment when trading against other fixed-mix rules, provided all agents use the same saving rate (see also Kelly 1956; Evstigneev et al. 2009).

Assuming R1-R2, we depart from the standard approach that derives consumption and portfolio decision from the maximization of an objective function. We also exclude rules that depend on market prices or on agents' wealth. Moreover, since beliefs and relative dividends are fixed, rules do not depend neither on the history of asset dividends nor on the price processes. The framework is, however, more general than what it might seem at first sight. First, our analysis also applies to the long-run behavior of markets where beliefs evolve over time, provided that beliefs converge to some constant level as more and more information is gathered. Second, it should be noted that Subjective Generalized Kelly portfolio (and saving) decisions of agent $i$ implied by $\mathbf{R} 1-\mathbf{R} 2$ coincide with those taken on an equilibrium path by a representative agent that maximizes a geometrically discounted log-utility with subjective beliefs $\pi^{i}$ and a discount factor equal to the saving rate $\delta^{i}$. As a result, the Subjective Generalized Kelly rule of agent $i$ is also optimal in an heterogeneous agents economy in the limit of

10 The same condition is assumed in the general equilibrium literature that investigates the Market Selection Hypothesis to guarantee existence of a competitive equilibrium (see, e.g., Axiom 3 in Blume and Easley 2006). 
agent $i$ holding all the aggregate endowment. Third, our analysis can be more generally applied to economies populated by agents with different limit risk preferences than an instantaneous log-utility, see the example in Sect. 5.4.

\section{Market dynamics}

In this section, we show that when agents use fixed-mix rules, intertemporal budget constraints (4) and market clearing conditions (5) can be solved to derive a unique sequence of positive and arbitrage-free equilibrium prices. Without loss of generality, we assume that each agent $i \in N$ starts with some given positive wealth $W_{0}^{i}{ }^{11}$

\subsection{Representative agent}

We start from the case where agent $i$ possesses all the aggregate endowment in $t=0$, so that $W_{0}^{j}=0$ for all $j \neq i$. Straightforward computations lead to

$$
\begin{gathered}
W_{t}^{i}=\frac{Y_{t}}{1-\delta^{i}}, \quad W_{t}^{j}=0, j \neq i, \quad \forall t \in \mathbb{N}_{0}, \\
P_{k, t}=\frac{\delta^{i} Y_{t}}{1-\delta^{i}} \mathrm{E}^{\pi^{i}}\left[d_{k}\right], \quad \forall k \in K, \quad \forall t \in \mathbb{N}_{0} .
\end{gathered}
$$

Asset $k$ is priced as in a log-economy where the representative agent has beliefs $\pi^{i}$ and discount factor equal to the saving rate $\delta^{i}$. The Lucas' model is recovered. In particular, if the dividend matrix is non-singular and the aggregate endowment is not risky, then risk neutral probabilities coincide with agent $i$ 's beliefs.

\subsection{Heterogeneous agents}

Pricing is more interesting when agents have heterogeneous beliefs. Assume that there exist at least two agents $i$ and $j$ with positive wealth and that $\alpha^{i} \neq \alpha^{j}$. Given investment rules, the initial price vector $P_{0}$ can be found from the initial wealth distribution $W_{0}$ using the market clearing condition (5). To find prices for $t \geq 1$, substitute (4) in (5) and obtain

$$
\sum_{l=1}^{K}\left(\mathbb{I}_{k, l}-\sum_{i=1}^{N} \frac{\alpha_{k}^{i} \alpha_{l}^{i} W_{t-1}^{i}}{P_{l, t-1}}\right) P_{l, t}=\sum_{l=1}^{K} d_{l}\left(s_{t}\right) Y_{t} \sum_{i=1}^{N} \frac{\alpha_{k}^{i} \alpha_{l}^{i} W_{t-1}^{i}}{P_{l, t-1}}, \quad \forall k \in K
$$

The above expression can be conveniently written in matrix form. Consider the vectors of price-rescaled investment fractions,

11 This is implied by assuming that agents start with an initial allocation of assets and consumption good. 


$$
\beta^{i}(W ; \alpha)=\left(\frac{\alpha_{1}^{i}}{\sum_{j=1}^{N} W^{j} \alpha_{1}^{j}}, \ldots, \frac{\alpha_{K}^{i}}{\sum_{i=j}^{N} W^{j} \alpha_{K}^{j}}\right), \quad \forall k \in K
$$

and define the positive matrix

$$
A(W ; \alpha)=\sum_{i=1}^{N} W^{i} \alpha^{i} \otimes \beta^{i}(W, \alpha)
$$

Equation (8) becomes

$$
\left(\mathbb{I}-A\left(W_{t-1} ; \alpha\right)\right) P_{t}=A\left(W_{t-1} ; \alpha\right) d\left(s_{t}\right) Y_{t}
$$

An intermediate step is to show that $\mathbb{I}-A(W ; \alpha)$ is invertible.

Lemma 1 Under the assumption that rules satisfy $\mathbf{R} \mathbf{1}-\mathbf{R} 2$, the matrix $\mathbb{I}-A(W, \alpha)$ is invertible for all $W \in \mathbb{R}_{+}^{N}$.

From the previous Lemma and from (10), it follows that market clearing prices are uniquely defined for every $t \geq 1$ and given by

$$
\begin{aligned}
P_{t}\left(\sigma_{t}\right) & =\left(\mathbb{I}-A\left(W_{t-1} ; \alpha\right)\right)^{-1} A\left(W_{t-1} ; \alpha\right) d\left(s_{t}\right) Y_{t}\left(\sigma_{t}\right) \\
& =\sum_{n=1}^{\infty} A^{n}\left(W_{t-1} ; \alpha\right) d\left(s_{t}\right) Y_{t}\left(\sigma_{t}\right) .
\end{aligned}
$$

Given D3 and R2, the series expansion implies that prices are positive. Date $t$ prices depend both on $W_{t-1}$, the previous date wealth distribution, and on $d\left(s_{t}\right) Y_{t}$, the realization of the dividend process. The above equation with D1 and D2 also implies that there exists a matrix $P(W ; \alpha, D)$, with the same dimension of $D$, such that $P_{k, s_{t}}\left(W_{t-1} ; \alpha, D Y_{t}\right)=P_{k, t}\left(\sigma_{t}\right)$. When the wealth distribution is degenerate, in that only agent $i$ has positive wealth, it is $A=\alpha^{i} \otimes \mathbf{1}$ and (7) is recovered. Long-lived asset prices and dividends $D$ determine the payoff matrix

$$
R(W ; \alpha, D)=P(W ; \alpha, D)+D=(\mathbb{I}-A(W ; \alpha))^{-1} D
$$

Through prices, payoffs depend on the wealth distribution $W$ and keep changing as the wealth distribution evolves. By substituting (12) in (4), one obtains the explicit evolution of the wealth distribution. By construction, it is adapted to the information filtration.

Under the standard utility maximization approach with unconstrained portfolios, arbitrage never occurs in equilibrium. In our model, however, asset holdings are constrained by $\mathbf{R} 1$ and arbitrage might occur. A sufficient condition to avoid arbitrages turns out to be that the vector of portfolio rules is in the interior of the cone generated by the $S$ columns of the matrix $D$ (see the proof of the Proposition 1). Given R1-R2, the condition is naturally satisfied by Subjective Generalized Kelly rules.

We summarize the results of this section in the following Proposition. 
Proposition 1 Consider an exchange economy where $N$ agents using rules obeying R1-R2 are trading $K$ assets satisfying D1-D3. If $W_{0} \in \mathbb{R}_{++}^{N}$, then for all $t \in \mathbb{N}$, $W_{t} \in \mathbb{R}_{++}^{N}$, is adapted to $\Im_{t}$, and evolves according to

$$
\begin{aligned}
W_{t}^{i}\left(\sigma_{t}\right)= & W_{t-1}^{i}\left(\sigma_{t-1}\right) \sum_{k=1}^{K} \beta_{k}^{i}\left(W_{t-1}\left(\sigma_{t-1}\right) ; \alpha\right) \\
& R_{k, s_{t}}\left(W_{t-1}\left(\sigma_{t-1}\right) ; \alpha, D Y_{t}\left(\sigma_{t}\right)\right), \quad \forall i \in N .
\end{aligned}
$$

Moreover, the sequence of wealth distributions $\left\{W_{t}\right\}$ is such that for all $t \in \mathbb{N}$ market equilibrium prices $P_{t}=P_{s_{t}}\left(W_{t-1} ; \alpha, D Y_{t}\right)$ and payoffs $R_{t+1}=R\left(W_{t} ; \alpha, D Y_{t+1}\right)$ do not admit arbitrage.

The first part of Proposition 1 corresponds to Proposition 1 in Evstigneev et al. (2006, 2008). ${ }^{12}$ In addition, we derive an explicit solution for equilibrium prices $P$ and asset payoffs $R$. This, in turn, allows us to prove that requiring agents to use Subjective Generalized Kelly portfolios, R1-R2, is sufficient to avoid arbitrages.

\subsection{Relative wealth dynamics}

If agents have different saving rates, the agent who saves more is advantaged in terms of wealth accumulation. If, for example, there are two agents $i=1,2$ with the same portfolio rule but $\delta^{1}>\delta^{2}$, then the wealth of agent 1 grows geometrically faster than the wealth of agent 2 , with rate $\delta^{1} / \delta^{2}$. When agents have different portfolios, there is a trade-off between having a higher saving rate and a "better" portfolio. Although the trade-off is certainly interesting, here we concentrate on the heterogeneity of portfolio rules and assume homogeneous saving rates:

$\mathbf{R 3} \delta^{i}=\delta, \forall i \in N$.

We shall show that under $\mathbf{R 3}$ the relative wealth dynamics does not depend on the aggregate endowment process. Normalized wealth and prices are

$$
w_{t}^{i}=\frac{1-\delta}{Y_{t}} W_{t}^{i}, \quad \forall i \in N, \quad \text { and } \quad p_{k, t}=\frac{1-\delta}{\delta Y_{t}} P_{k, t}, \quad \forall k \in K
$$

so that, for any $t, \sum_{i=1}^{N} w_{t}^{i}=1$ and $\sum_{k=1}^{K} p_{k, t}=1$. Lemma 1 and Proposition 1 can be restated in terms of normalized variables by replacing date $t$ payoffs $R_{t}$ with their normalizations by the total wealth $Y_{t} /(1-\delta)$. As a result, the payoff matrix $R$ defined in (12) becomes

$$
r(w ; x, \delta, D)=(1-\delta)(\mathbb{I}-\delta A(w ; x))^{-1} D,
$$

\footnotetext{
12 Evstigneev et al. (2006) establish the result in the more general case of adapted portfolio rules. It is straightforward to see that our proof holds even when beliefs $\pi_{t}^{i}$ are adapted to the information filtration generated by $s_{\tau}$ and $P_{\tau-1}$ for all $\tau \leq t$.
} 
where the matrix $A$ depends on the normalized wealth and portfolio shares $x$. In particular, the payoff of asset $k$ when state $s$ is realized can be written as a convex combination of relative dividends and normalized prices:

$$
r_{k, s}(w ; x, \delta, D)=(1-\delta) d_{k, s}+\delta p_{k, s}(w ; x, \delta, D)
$$

When $\delta$ is close to zero, the contribution of normalized prices becomes very small and the normalized payoff matrix $r$ approaches the dividend matrix $D$. This is because in our economy equilibrium prices can be written as the discounted sum of future dividends, see (11). As a result, when the saving rate is close to zero, prices become small too. Conversely, when $\delta$ is close to one, prices are much larger than dividends. In the limit $\delta \rightarrow 1$, dividends do not count and the payoff matrix becomes singular.

An advantage of working with normalized variables is that, since states of the world are i.i.d. and agents agree on that, the relative wealth dynamics is a Markov process.

Corollary 1 Under the assumptions of Proposition 1, if saving rules obey to $\mathbf{R} \mathbf{3}$, then the normalized wealth $w_{t}$ follows a Markov Process on $\Delta_{+}^{N}$ such that, for every $t \in \mathbb{N}$, with probability $\pi(s)$ the relative wealth vector $w_{t-1}$ evolves into

$$
w_{t}^{i}=w_{t-1}^{i} \sum_{k=1}^{K} \beta_{k}^{i}\left(w_{t-1} ; x\right) r_{k, s}\left(w_{t-1} ; x, \delta, D\right), \quad \forall i \in N
$$

In the rest of the paper, we shall use (15) to study the dynamics of the relative wealth of groups of agents.

\section{Market selection and long-run heterogeneity}

Let $I \subset N$ be a proper subset of agents and $I^{c}=N \backslash I$ its complement. We denote with $w_{t}^{I}$ the sum of date- $t$ wealth of agents in $I$, so that $1-w_{t}^{I}$ is the sum of date- $t$ wealth of agents in $I^{c}$. The aggregate portfolio rule of group $I$ is

$$
x^{I}\left(w_{t} ; x\right)=\sum_{i \in I} x^{i} \frac{w_{t}^{i}}{w_{t}^{I}}
$$

while its rescaled portfolio is

$$
\beta^{I}\left(w_{t} ; x\right)=\left(\frac{x_{1}^{I}\left(w_{t} ; x\right)}{\sum_{i=1}^{N} x_{1}^{i} w_{t}^{i}}, \ldots, \frac{x_{K}^{I}\left(w_{t} ; x\right)}{\sum_{i=1}^{N} x_{K}^{i} w_{t}^{i}}\right)
$$

In this section, we provide sufficient conditions for the survival or dominance of a generic group $I$. The next definition clarifies what we mean by dominance, survival, and vanishing. 
Definition 1 We say that group $I$ dominates on a sequence $\sigma$ if

$$
\lim _{t \rightarrow \infty} w_{t}^{I}(\sigma)=1
$$

Group $I$ survives on a sequence $\sigma$ if

$$
\limsup _{t \rightarrow \infty} w_{t}^{I}(\sigma)>0
$$

If a group does not survive on $\sigma$, we say that it vanishes on that sequence. We say that group I dominates or survives if (17) or (18) holds P-a.s. Group I vanishes if it does not survive.

If a group composed by a single agent dominates, heterogeneity is a transient property and the economy converges with probability one to its representative agent limit. If instead more than one agent survives, then the economy exhibits long-run heterogeneity.

Definition 2 An $N$-agent asset market economy exhibits long-run heterogeneity if there exists a proper subset of traders $I \subset N$ such that both the group $I$ and the group $I^{c}$ survive.

In order to characterize the relative performance of group $I$, we use the difference between the conditional expected log wealth growth rate of group $I$ and the conditional expected log wealth growth rate of its complement $I^{c}$, that is, the conditional drift of the process $\log \left(w_{t}^{I} / w_{t}^{I^{c}}\right)$. Corollary 1 implies that this quantity depends on $\Im_{t}$ only through the date- $t$ normalized wealth distribution, formally

$$
\begin{aligned}
\mathrm{E}^{\mathrm{P}}\left[\log \frac{w_{t+1}^{I}}{w_{t}^{I}}-\log \frac{1-w_{t+1}^{I}}{1-w_{t}^{I}} \mid \Im_{t}\right] & =\mathrm{E}^{\pi}\left[\log \frac{\sum_{k=1}^{K} \beta_{k}^{I}\left(w_{t} ; x\right) r_{k}\left(w_{t} ; x, \delta, D\right)}{\sum_{k=1}^{K} \beta_{k}^{I^{c}}\left(w_{t} ; x\right) r_{k}\left(w_{t} ; x, \delta, D\right)}\right] \\
& =\mu^{I}\left(w_{t}\right)
\end{aligned}
$$

The sign of $\mu^{I}\left(w_{t}\right)$ tells us whether, in relative terms, the aggregate wealth of agents in $I$ grows or shrinks, in expectation, with respect to the aggregate wealth of agents in $I^{c}$. It turns out that one can derive sufficient conditions for survival or dominance of a group by studying the sign of $\mu^{I}\left(w_{t}\right)$ when the relative wealth $w_{t}^{I}$ is very large or very small. For a proper subset $I$ and for $v \in[0,1]$, define

$$
\bar{\mu}^{I}(v)=\max \left\{\mu^{I}(w) \quad \mid \quad w \in \Delta^{N}, w^{I}=v\right\},
$$

and

$$
\underline{\mu}^{I}(v)=\min \left\{\mu^{I}(w) \quad \mid \quad w \in \Delta^{N}, w^{I}=v\right\}
$$

The definition is meaningful because the function $\mu^{I}$ is continuous in $w$ and the extrema are computed on compact sets. Since the map between $v$ and these sets is 
continuous in $v$ (both upper and lower hemicontinuous), $\mu^{I}$ and $\bar{\mu}^{I}$ are also continuous function of $v$.

The next Proposition exploits the Martingale Converge Theorem (see the proposition's proof for details) to characterize long-run survival. Intuitively, if the expected growth rate of a group of agents is positive when its aggregate wealth is sufficiently small and under the most adverse wealth distribution of the other group, then the group cannot vanish.

Proposition 2 Consider an exchange economy with $N$ agents using rules obeying R1-R3 and trading $K$ assets satisfying D1-D4:

(i) If $\mu^{I}(0)>0$, then group I survives.

(ii) If $\bar{\mu}^{I}(1)<0$, then group $I^{c}$ survives.

If the inequality on bounds of the conditional drift stated in Proposition 2 holds, then, a forziori, it holds also for the conditional drift computed along (almost) all possible trajectories of our economy. On the other hand, the Proposition provides only a sufficient condition, because, by considering the extremal drift values over all possible wealth distributions, we might consider distributions that cannot be realized with positive probability. Under (i) and (ii), both groups $I$ and $I^{c}$ survive and the market exhibits long-run heterogeneity. In order to derive other results, such as dominance of a group, we need to assume that group $I$ aggregate rule $x^{I}$ cannot be replicated by a combination of other agents' rules. Specifically we assume that

R4 There exists a hyper-plane in $\mathbb{R}^{K}$ which separates the rules of agents in $I$ from the rules of agents in $I^{c}$.

Since the aggregate rules $x^{I}$ and $x^{I^{c}}$ belong to the convex cone generated by the strategies of agents in $I$ and $I^{c}$, respectively, condition $\mathbf{R 4}$ is sufficient to guarantee that they can never be equal. When individual rules are all different and there are at least as many assets as agents, $K \geq N$, condition $\mathbf{R} 4$ is satisfied for any group $I$.

Assumption R4, combined with the absence of redundant assets D4, is sufficient to prove that, for all $t$, there is a positive probability that the wealth distribution between group $I$ and group $I^{c}$ keeps changing.

Lemma 2 Under $\mathbf{R} 4$, if there are no redundant assets, $\mathbf{D 4}$, then there exists a $\gamma>0$ such that for all $t \in \mathbb{N}_{0}$

$$
\operatorname{Prob}\left\{\left|\log \frac{w_{t+1}^{I}}{w_{t+1}^{I^{c}}}-\log \frac{w_{t}^{I}}{w_{t}^{I^{c}}}\right|>\gamma \mid \Im_{t}\right\}>\gamma \text {. }
$$

Thus, as long as the two groups have different rules, no constant wealth distribution is possible. Under the same hypothesis, plus lack of arbitrage, we can also rule out the possibility that the conditional wealth growth rate of one group is larger than the other with full probability. 
Lemma 3 Under R4, if market equilibrium prices $p_{t}$ and asset payoffs $r_{t+1}$ do not admit arbitrages, then for all groups $I$ there exists an $\epsilon>0$ such that for all $t \in \mathbb{N}_{0}$

$$
\operatorname{Prob}\left\{\frac{w_{t+1}^{I}}{w_{t}^{I}}>\frac{w_{t+1}^{I^{c}}}{w_{t}^{I^{c}}} \mid \Im_{t}\right\}>\epsilon \text { and } \operatorname{Prob}\left\{\frac{w_{t+1}^{I}}{w_{t}^{I}}<\frac{w_{t+1}^{I^{c}}}{w_{t}^{I^{c}}} \mid \Im_{t}\right\}>\epsilon
$$

Using asymptotic conditions on the sign of groups' wealth growth rates, and based on the previous two Lemmas, we can obtain sufficient conditions for a group to dominate or vanish.

Proposition 3 Consider an exchange economy with $N$ agents using rules obeying R1-R4 and trading $K$ assets satisfying D1-D4:

(i) If $\mu^{I}(0)>0$ and $\mu^{I}(1)>0$, then group I dominates;

(ii) If $\overline{\bar{\mu}}^{I}(0)<0$ and $\overline{\bar{\mu}}^{I}(1)<0$, then group I vanishes.

(iii) If $\mu^{I}(0)>0$ and $\bar{\mu}^{I}(1)<0$, then both groups $I, I^{c}$ survive and for $G=I, I^{c}$

$$
\operatorname{Prob}\left\{\liminf _{t \rightarrow \infty} w_{t}^{G}=0 \text { and } \limsup _{t \rightarrow \infty} w_{t}^{G}=1\right\}=1
$$

When both groups survive, case (iii), Proposition 3 complements Proposition 2 by clarifying that, under R4 and lack of arbitrage, groups' wealth shares keep fluctuating in the interval $(0,1)$. Asset prices fluctuate accordingly. Case (i), and symmetrically (ii), says that a group dominates if it performs, on average, better than its complement both in the limit when its wealth is small and in the limit when it is large. The separability of rules $\mathbf{R} \mathbf{4}$ and the lack of arbitrage are required to ensure that relative wealth ratios keep changing. In particular, they forbid the innovations of the process $\left\{\log \left(w_{t}^{I} /\left(1-w_{t}^{I}\right)\right)\right\}$ from having a definite sign. In fact, even if the asymptotic drift conditions say that group $I$ has better performances both when it is large and when it is small, the presence of a limited arbitrage in favor of group $I^{c}$ when $w \in(0,1)$ could prevent group $I$ from exploiting its asymptotic advantage.

As a Corollary of Proposition 3, we are able to establish that if agent has correct beliefs, then she dominates.

Corollary 2 Under the assumptions of proposition 3, if agent $i$ has correct beliefs, $\pi^{i}=\pi$, then she dominates.

This result is also implied by Theorem 5 in Evstigneev et al. (2008), which is more generally proved without requiring that other agents' fixed-mix rules are Subjective Generalized Kelly, R1-R2, nor separated, R4. However, our proof relies only on the sign of asymptotic drifts.

\subsection{Two-agent economy}

In this section, we characterize the long-run outcomes of a two-agent economy and show that sufficient conditions of Propositions 2-3 become tight. Most of the examples in Sect. 5 shall exploit these conditions to provide a full characterization of the possible long-run outcomes in specific two-agent economies. Consider $N=\{1,2\}$. Given 
wealth normalizations, the conditional drift (19) can be written as function of $w_{t}^{1}$ only

$$
\mathrm{E}^{\pi}\left[\log \frac{\sum_{k=1}^{K} \beta_{k}^{1}(w ; x) r_{k}(w ; x, \delta, D)}{\sum_{k=1}^{K} \beta_{k}^{2}(w ; x) r_{k}(w ; x, \delta, D)}\right]=\mu\left(w_{t}^{1}\right)
$$

Moreover, for all $v \in[0,1], \mu^{1}(v)=\bar{\mu}^{1}(v)=\mu(v)$. In what follows, it is convenient to define the relative entropy, or Kullback-Leibler divergence, of rules $x^{i}$ with respect to the reference rule $x^{*}$ with $x^{*}=\mathrm{E}^{\pi}[d]$ as

$$
K L\left(x^{i} \| x^{*}\right)=\mathrm{E}^{x^{*}}\left[\log \left(\frac{x^{*}}{x^{i}}\right)\right] .
$$

The difference of relative entropies is

$$
\nabla_{x^{*}}\left(x^{2} \| x^{1}\right)=K L\left(x^{2} \| x^{*}\right)-K L\left(x^{1} \| x^{*}\right) .
$$

The following lemma provides an ordering of asymptotic drifts with respect to the difference of rules' relative entropies.

Lemma 4 Consider an exchange economy with $N=2$ agents using rules obeying $\mathbf{R 1}-\mathbf{R} 4$ and trading $K$ assets satisfying $\mathbf{D 1}-\mathbf{D 4}$, then

$$
\mu(0)>(1-\delta) \nabla_{x^{*}}\left(x^{2}|| x^{1}\right)>\mu(1) .
$$

The Lemma establishes that, in relative terms, an agent is better off in the limit of her wealth share being 0 than in the limit of her wealth share being 1 . In particular, if an agent has a favorable drift when her wealth share approaches 1, i.e., she has a higher expected log wealth growth rate than the other agent, then she has also a favorable drift in the limit of her wealth share approaching 0. Conversely, if an agent faces an unfavorable drift in the limit of her wealth share approaching 0 , then the drift is unfavorable also in the limit of her wealth share approaching 1. In Sect. 5.1, we shall use the notion of effective beliefs to provide an intuition behind the Lemma.

Coupling the previous result with Proposition 3 leads to the following set of sufficient and generically necessary conditions characterizing long-run outcomes of a two-agent economy.

Proposition 4 Consider an exchange economy with $N=2$ agents using rules obeying R1-R4 and trading $K$ assets satisfying D1-D4. Provided both $\mu(0)$ and $\mu(1)$ have a definite sign, one of the following occurs

(i) If $\mu(1)>0$, then agent 1 dominates and 2 vanishes;

(ii) If $\mu(0)<0$, then agent 2 dominates and 1 vanishes;

(iii) If $\mu(0)>0$ and $\mu(1)<0$, then both agents survive and for all assets $k \in K$

$$
\operatorname{Prob}\left\{\liminf _{t \rightarrow \infty} p_{k, t}=\min _{i=1,2}\left\{\mathrm{E}^{\pi^{i}}\left[d_{k}\right]\right\} \text { and } \limsup _{t \rightarrow \infty} p_{k, t}=\max _{i=1,2}\left\{\mathrm{E}^{\pi^{i}}\left[d_{k}\right]\right\}\right\}=1 .
$$


According to Lemma 4, and leaving out non-generic situations in which asymptotic drifts are zero, no other cases other than those covered by Proposition 4 can occur. Moreover, contrary to the bound of conditional drifts in Propositions 2 and 3, $\mu(0)$ and $\mu(1)$ can be computed easily, making the two-agent economy particularly tractable and amenable to applications. Having $\mu(1)>0(\mu(0)<0)$ is sufficient to prove that agent 1 (2) dominates. When $\mu(0)>0$ and $\mu(1)<0$, both agents 1 and 2 survive and none dominates. ${ }^{13}$ In other terms, long-run heterogeneity occurs when both agents have higher wealth growth rates at the returns determined by the other agent. As in Proposition 3, long-run heterogeneity amounts to a relative wealth that does not converge and to prices that keep fluctuating between the two agents' evaluations. In the next section, we prove that such cases do always exist and are robust to perturbations of agents' beliefs.

Lemma 4 and Proposition 4 imply the survival of the agent whose portfolio rule is the closest, in terms of relative entropy, to $x^{*}$, the Generalized Kelly rule of Evstigneev et al. (2008). This extends the result of Bektur (2013) who shows that if a rule is, coordinate-wise, the closest to $x^{*}$ then it survives. The extension concerns two dimensions. Firstly, we make clear that the appropriate "distance" of rules is the relative entropy with respect to the Generalized Kelly portfolio. Secondly, we show that whether a specific agent also dominates, or, conversely, both agents survive, can be stated by studying the limits in which they have, respectively, all the wealth. As also shown by Corollary 2, the Generalized Kelly trader dominates against all possible Subjective Generalized Kelly traders. Summarizing, we have the following.

Corollary 3 Consider an exchange economy with $N=2$ agents using rules obeying R1-R4 and trading $K$ assets satisfying D1-D4. If agents' beliefs are such that

$$
K L\left(x^{2} \| x^{*}\right)>K L\left(x^{1} \| x^{*}\right)
$$

then agent 1 survives. If, moreover, agent 1 's beliefs are correct, so that $x^{1}=x^{*}$, then agent 1 dominates.

In particular, when $D=\mathbb{I}$, the above inequality can be written in terms of beliefs and becomes

$$
K L\left(\pi^{2}|| \pi\right)>K L\left(\pi^{1} \| \pi\right) .
$$

In this case, agent 1 survives when she has more accurate beliefs and having correct beliefs is always sufficient for dominance. With more general dividend matrices $D$ and, possibly, incomplete markets, it is the relative entropy of rules, rather than of beliefs, that guarantees survival to the most accurate rule.

\footnotetext{
13 A fourth outcome that might occur in market selection models is path dependency, which arises when each rule has a favorable drift in the limit when it has all the wealth. Horst and Wenzelburger (2008) find path dependency when two competing financial mediators are selected by investors. Bottazzi and Dindo (2014) find path dependency when rules are allowed to depend on prices.
} 


\subsection{Long-run heterogeneity}

Having derived sufficient conditions for long-run heterogeneity, we turn to generality and existence. Firstly, when long-run heterogeneity occurs, it is also generic: Perturbations of beliefs do not lead to dominance of any of the surviving agent. The sufficient conditions for long-run heterogeneity involve strict inequalities. Since the conditional drift $\mu\left(w^{1}\right)=\mu\left(w^{1} ; \pi^{1}, \pi^{2}\right)$ is a continuous functions of beliefs (via the portfolio rules), then it is possible to perturb the latter without violating the inequalities. Formally

Corollary 4 If in an economy as in Proposition 4 the beliefs $\pi^{1}, \pi^{2}$ are such that (iii) is satisfied, then there exist vectors $\epsilon^{1}, \epsilon^{2} \in \mathbb{R}^{S}$ with components $\epsilon_{s}^{i} \in[-\varepsilon, \varepsilon], \varepsilon>0$, and $\sum_{s=1}^{S} \epsilon_{s}^{i}=0$ for $i=1,2$, such that under beliefs $\pi^{1}+\epsilon^{1}$ and $\pi^{2}+\epsilon^{2}$ condition (iii) is still satisfied.

Secondly, we show that for any asset structure $D$ there exist beliefs for which heterogeneity is indeed the long-run outcome. We restrict our analysis to an economy with $N=2$ and assume that both agents do not know the truth, $\pi^{i} \neq \pi$ for $i=1,2$. The result of Lemma 4 together with condition (iii) of Proposition 4 implies that if two agents have beliefs such that the corresponding rules have the same relative entropy with respect to $x^{*}$, then they both survive. Thus, in order to prove the existence of long-run heterogeneity for every admissible choice of the matrix $D$, we have to find beliefs for agents 1 and 2 such that the corresponding Subjective Generalized Kelly rules have the same relative entropy. For this purpose, define $\bar{\Delta}$ as the open set of all possible Subjective Generalized Kelly portfolios given the dividend matrix $D$, $\bar{\Delta}=\left\{x^{\prime} \in \Delta^{K}: x^{\prime}=\mathrm{E}^{\pi^{\prime}}[D], \pi^{\prime} \in \Delta_{+}^{S}\right\}$, and call $\partial(\bar{\Delta})$ its frontier.

Proposition 5 Given a dividend matrix D and beliefs $\pi^{1} \neq \pi$ such that $K L\left(x^{1} \| x^{*}\right)<$ $\mathcal{K}$ with $\mathcal{K}=\min _{\pi^{\prime}}\left\{K L\left(x^{\prime} \| x^{*}\right)\right.$ s.t. $\left.x^{\prime} \in \partial(\bar{\Delta})\right\}$, there exists a non-empty set of beliefs $\Pi \subset \Delta_{+}^{S}$ with $\pi^{1} \in \Pi$ such that for all $\pi^{2} \in \Pi$ the asset market economy with Subjective Generalized Kelly traders having subjective beliefs $\pi^{1}$ and $\pi^{2}$ exhibits long-run heterogeneity.

The fundamental ingredients for proving Proposition 5 are the properties of the relative entropy. Its continuity, strict convexity, and the fact that it has a minimum equal to zero in $x^{*}$ are sufficient to show the existence of $\Pi$. Indeed, in order to build $\Pi$, it is enough to fix $\pi^{1}$ and take the set of beliefs such that the Subjective Generalized Kelly portfolio they generate have all the same relative entropy with respect to $x^{*} .{ }^{14}$ Then, thanks to Corollary 4 , one can expand the set by including neighborhoods of all these beliefs.

The equality of beliefs' relative entropy, or more generally the equality of a suitably defined survival index that mixes beliefs and discount factors, may imply long-run heterogeneity also in market economies where agents are expected utility maximizers and asset markets are complete (see, e.g., Blume and Easley 2009; Jouini and Napp 2010). There is, however, an important difference with the model presented here.

\footnotetext{
14 The fact that $\Pi \subset \Delta_{+}^{S}$ depends on the technical condition $K L\left(x^{1} \| x^{*}\right)<\mathcal{K}$. Otherwise, the set of rules with relative entropy equal to a given $x^{1}$ could encompass rules that are not generated by any belief.
} 
Whereas heterogeneity is generic in our market, see Corollary 4 , it is non-generic in the former literature. Indeed, any small perturbation of an agent's beliefs breaks the tie among survival indexes and thus leads to the dominance of a single agent.

\section{Discussion and examples}

We begin this section by providing an intuition for our results based on the comparison between Subjective Generalized Kelly rules and log-optimal rules. Then, we explore market selection outcomes in $\mathrm{N}$-agent economies for specific choices of the dividend matrix $D$. Finally, we consider an example where long-run heterogeneity occurs although every agent knows the truth.

\subsection{Effective beliefs}

In what follows, we introduce the concept of Effective Beliefs to provide an intuition behind the occurrence of long-run heterogeneity.

In a similar asset market economy, if portfolios are log-optimal and the asset market is dynamically complete, then the agent with the most accurate beliefs dominates (see, e.g., Sandroni 2000; Yan 2008). However, we find that when agents use Subjective Generalized Kelly rules the accuracy of beliefs is not directly related to dominance. Provided $D$ is diagonal, Corollary 3 proves only a weaker result, that is, accuracy of beliefs is sufficient for survival. Agent $i$ 's Subjective Generalized Kelly portfolio might not dominate, in spite of agent $i$ having more accurate beliefs than the other agent and using a subjectively log-optimal portfolio, because the portfolio of the agent with less accurate subjective beliefs might be closer to the (objectively) log-optimal portfolio when agent $i$ 's holds all the wealth. Non-accuracy of beliefs and non-log-optimality of the portfolio must compensate each others.

In order to establish how, and when, this compensation occurs, we introduce the concept of effective beliefs. Given asset prices in $t$ and payoffs in $t+1$, we define agent $i$ 's effective beliefs in $t, \hat{\pi}_{t}^{i}$, as the beliefs such that the Subjective Generalized Kelly rule $x^{i}$ derived from $\pi^{i}$ is log-optimal in $t$. More specifically, to compute effective beliefs we proceed as follows. Given agents' beliefs, saving rates, and a dividend matrix $D$, for every value of the relative wealth distribution $w_{t}$ there correspond both a vector of prices $p_{t}$ and a payoff matrix $r_{t+1}$ (see Sect. 3.3 for details). Thus, for every $w_{t}$ one can define the effective beliefs of agent $i$ as those beliefs $\hat{\pi}_{t}^{i}$ such that her portfolio rule $x^{i}$ is (subjectively) log-optimal given prices $p_{t}$ and payoffs $r_{t+1}$. As a result, for each agent $i$, we derive a function $\hat{\pi}^{i}: \Delta^{N} \rightarrow \Delta_{+}^{S}$ such that $\hat{\pi}_{t}^{i}(s)=\hat{\pi}_{s}^{i}\left(w_{t} ;\left\{\pi^{j}\right\}, \delta, D\right), \forall s \in S$. Note that the function depends on all agents' beliefs $\left\{\pi^{j}\right\}$, on their saving rate $\delta$, and on the dividend matrix $D$. It is so because all these quantities contribute to determine the payoff matrix in equilibrium. Given the (subjective) log-optimality of the Subjective Generalized Kelly rule when she has all wealth, it is $\hat{\pi}^{i}\left(\left(0, \ldots, w^{i}=1, \ldots, 0\right) ;\left\{\pi^{j}\right\}, \delta, D\right)=\pi^{i}$ for all $i \in N$, independently from $\delta, D$, and other agents' beliefs.

This construction enables us to view the economy with Subjective Generalized Kelly traders with beliefs $\left\{\pi^{i}\right\}$ as an economy with (subjective) log-optimal traders 
using effective beliefs $\left\{\hat{\pi}_{t}^{i}\right\}$. The general equilibrium literature tells us that, provided the asset market is complete, an agent survives only when her beliefs are, on average, as accurate as prices (see Massari 2017). As a result, whenever we find that long-run heterogeneity is the long-run outcome, agents' effective beliefs must be, on average, equally accurate. Moreover, along the lines of Propositions 3 and 4, one can prove that the sufficient conditions that characterize long-run outcomes can be given in terms of asymptotic effective beliefs accuracy instead that in terms of asymptotic growth rates $\mu$. In fact, the following proposition shows that the relative accuracy of effective beliefs can be used to characterize the value of asymptotic drifts $\mu(0)$ and $\mu(1) .{ }^{15}$

Proposition 6 Consider an exchange economy with $N=2$ agents using rules obeying R1 - R4 and trading a complete set of assets satisfying D1 - D4 with non-singular dividend matrix $D$, then

$$
\begin{aligned}
& \mu(0)=\nabla_{\pi}\left(\pi^{2} \| \hat{\pi}^{1}\left((0,1) ; \pi^{1}, \pi^{2}, \delta, D\right)\right) \text { and } \\
& \left.\mu(1)=\nabla_{\pi}\left(\hat{\pi}^{2}\left((1,0) ; \pi^{1}, \pi^{2}, \delta, D\right)\right) \| \pi^{1}\right) .
\end{aligned}
$$

Together with Proposition 4, the former implies that in a two-agent economy, longrun heterogeneity occurs when, for both $i=1,2$, agent $i$ 's effective beliefs are more accurate than agent $j \neq i$ effective beliefs when agent $j$ sets prices and payoffs.

The market completeness assumption is important for two reasons. First, only when markets are complete, it is possible to uniquely determine effective beliefs by agents' portfolio positions, asset payoffs, and asset prices. Second, only when markets are complete, selection of expected utility maximizers with homogeneous discount factors favors accurate beliefs (see, e.g., Blume and Easley 2006). Thus, only when markets are complete, survival of an agent with inaccurate beliefs and a non-optimal portfolio rule implies that the non-optimality of her rule must compensate the inaccuracy of her beliefs.

Figure 1 shows effective beliefs in a two-agent economy with complete markets, two states, and diagonal dividend matrix $D$. Effective beliefs depend on the value of $w^{1}$. By construction, effective beliefs and beliefs coincide when an agent has most of the wealth. However, beliefs and effective beliefs differ when both agents have positive wealth. In particular, given two agents, the effective beliefs of each agent are a combination of her beliefs with the beliefs of the other agent. The larger the wealth share of one agent, the larger her impact on equilibrium payoffs, the larger the weight of her beliefs in determining both agents' effective beliefs. This behavior of effective beliefs is consistent with the fact that for the same rule, say $x^{1}$, to be optimal under different equilibrium prices (and thus wealth distributions), effective beliefs $\hat{\pi}^{1}$ should move in the direction of beliefs $\pi^{2}$ the more $x^{2}$ becomes optimal. The saving rate $\delta$ is also important because $\delta$ determines the relative importance of prices and dividends in the total payoff matrix, see equation (14).

In the example of Fig. 1, $S=2$, and agents' beliefs are on opposite sides with respect to the truth: $\pi=(0.5,0.5), \pi^{1}=(0.75,0.25), \pi^{2}=(0.4,0.6)$. Agent 2 's

15 The proposition generalizes to $N$-agent economies by taking all the possible combinations of the two groups' effective beliefs. 


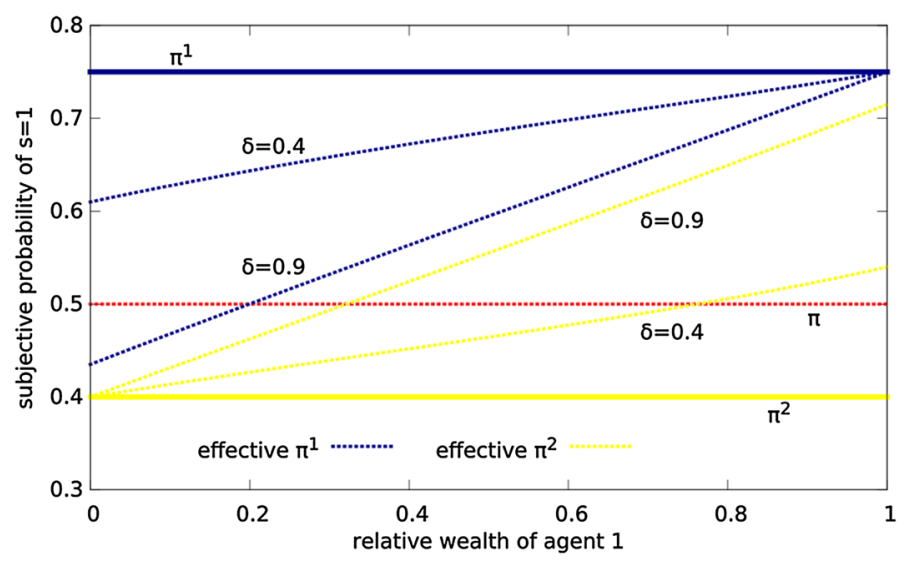

Fig. 1 Effective beliefs of two agents with different values of saving rate $\delta$ in a complete market of two assets with $D=\mathbb{I}$. Since the truth is $\pi=(1 / 2,1 / 2)$, the relative entropy is a symmetric function and the euclidean vertical distance between beliefs $\hat{\pi}^{i}$ and $\pi=(1 / 2,1 / 2)$ can be used directly to appraise $K L\left(\hat{\pi}^{i} \| \pi\right)$

beliefs are more accurate. Since $D$ is diagonal, by Corollary 3 agent 2 never vanishes. Whether agent 2 dominates or both agents survive depends on the saving rate, that is, on how much non-accuracy of beliefs and non-optimality of the Subjective Generalized Kelly rule influence each others. Simple calculations (and our numerical exploration of Sect. 5.2) show that agent 2 dominates when $\delta=0.4$ whereas both agents survive when $\delta=0.9$. Effective beliefs confirm this outcome. When $\delta=0.4$ agent 2 has better effective beliefs both when she has most of the wealth and when she has none, and thus dominates. In fact, she has most accurate effective beliefs for all possible wealth distributions. When $\delta=0.9$, however, each agent has most accurate effective beliefs when the other agent sets asset returns, so that both agents survive.

The graphical representation clarifies also why, for all $\delta \in(0,1)$, long-run heterogeneity is the long-run outcome when rules have the same relative entropy, see the proof of Proposition 5. Assume that the beliefs of agent 1 are $\pi^{1}(1)=0.6$ instead of $\pi^{1}(1)=0.75$, so that $\nabla_{\pi}\left(\pi^{2} \| \pi^{1}\right)=0$. Effective beliefs, by laying between the two agents beliefs, are such that the conditions for long-run heterogeneity are satisfied for all $\delta>0$.

Effective beliefs are also useful to provide an intuition for Lemma 4, that is, for the fact that $\mu(0)>\mu(1)$. Consider the representation of asymptotic drifts given in Proposition 6 and note that what counts for agents' relative performance, and thus for the drift of the relative wealth process, is the difference of relative entropies of effective beliefs. Moreover, in a two-agent economy, agents mutual influence through equilibrium prices is such that, component by component, agents' effective beliefs maintain the same ordering and have equal distance when one of the two agents dominates, as is also noted in Fig. 1. If it were the average euclidean distance of effective beliefs to determine asymptotic drifts, then $\mu(0)$ and $\mu(1)$ would be equal. However, due to the multiplicative nature of the wealth accumulation process, what 
counts is the average log-ratio of effective beliefs. As a result, an agent is better off, i.e., she earns relatively more or looses relatively less, when she copies the other agent (she is small) then when she is copied (she is large).

\subsection{Two-agent economies}

In what follows, we numerically explore the occurrence of long-run heterogeneity. We start with some examples of two-agent economies.

Diagonal assets Consider an economy with two states of the world where two Subjective Generalized Kelly agents trade two assets. Assume $D=\mathbb{I}$ and fix $\pi=(0.5,0.5)$. We use the conditions of Proposition 4 to characterize long-run outcomes for different values of the economy parameters.

In the upper plot of Fig. 2, the saving rate is $\delta=0.8$ and all possible combinations of agents' beliefs are considered. Given the two parameters $\pi_{1}^{1}$ and $\pi_{1}^{2}$, beliefs of agents 1 and 2 are, respectively, $\pi_{1}=\left(\pi_{1}^{1}, 1-\pi_{1}^{1}\right)$ and $\pi^{2}=\left(\pi_{1}^{2}, 1-\pi_{1}^{2}\right)$. Consistently with the behavior of effective beliefs, long-run heterogeneity occurs only for beliefs that are anti-correlated, that is, when one agent believes that asset 1 pays a dividend with probability greater than $1 / 2$ while the other believes the opposite. The figure is an example of the result of Corollary 3 for diagonal dividends matrices: The agent with beliefs farthest from the truth never dominates.

In the lower plot, we set $\pi^{2}=(0.6,0.4)$ and vary beliefs of agent 1 and the value of saving rate $\delta$. By determining the relative size of dividends and prices, $\delta$ affects the influence of the beliefs of one agent on the effective beliefs of the other. Notice that the area of long-run heterogeneity shrinks for low values of $\delta$ until it disappears when $\delta=0$. In fact, in the limit $\delta \rightarrow 0$, effective beliefs coincide with beliefs for all values of the wealth distribution so that log-run heterogeneity is only a non-generic phenomenon that takes place when beliefs have the same relative entropy with respect to the truth, $K L\left(\pi^{1} \| \pi\right)=K L\left(\pi^{2} \| \pi\right)$.

To give an idea of how a particular trajectory of wealth and prices looks like under long-run heterogeneity, we fix $\delta=0.8, \pi=(0.5,0.5), \pi^{1}=(0.45,0.55), \pi^{2}=$ $(0.6,0.4)$, and $w_{0}=0.5$. In the left panel of Fig. 3, we plot the evolution of wealth shares and the dynamics of asset 1's normalized price for $T=1000$ periods. As soon as the wealth share of an agent approaches low values, it bounces back so that, eventually, wealth shares are re-balanced. The normalized price of asset 1 follows a symmetric pattern, since, with two agents, it is a linear function of agent 1's wealth share.

The right panel of Fig. 3 shows the time histogram of asset 1's normalized price computed over 100,000 periods. As one can notice the distribution lies between the two agents' evaluations, as stated in point (iii) of Proposition 4.

Binomial Tree Consider now the case of the binomial tree economy of Sect. 2.1 with $r=g_{d} / g_{u}=0.2, \pi=(0.5,0.5)$. As before we can use our conditions to establish what happens for all the possible combinations of beliefs when the saving rate is $\delta=0.8$, upper panel of Fig. 4 , and for all possible combinations of $\delta$ and agent 1 beliefs when $\pi^{2}=(0.6,0.4)$, lower panel of Fig. 4 . 
Fig. 2 Areas of dominance and survival in an asset market economy with $D=\mathbb{I}$ as a function of beliefs $\pi^{1}$ and $\pi^{2}$ (upper panel) and saving rate $\delta$ and $\pi^{1}$ (lower panel). 1D: agent 1 dominates; $2 D$ : agent 2 dominates; $H$ : long-run heterogeneity (color figure online)
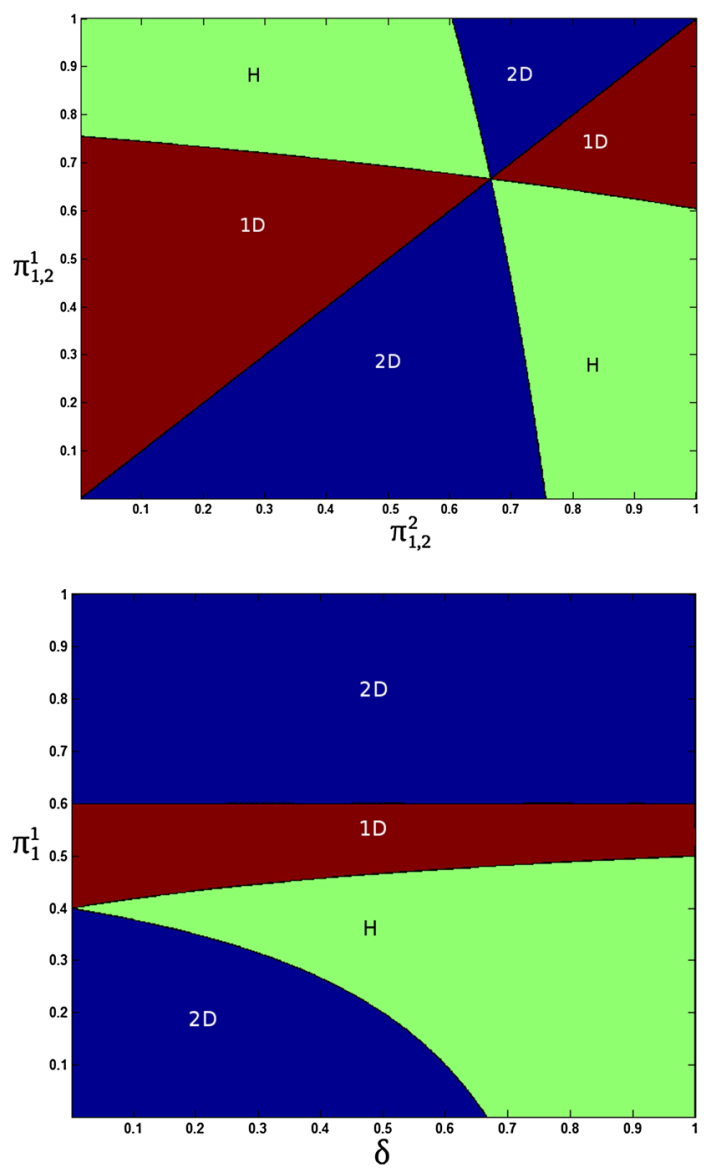

The characterization of the possible long-run outcomes is quite similar to the previous example, the only difference being that areas of long-run heterogeneity slightly increase. For example, with non-diagonal assets there exist cases of long-run heterogeneity even in the limit of $\delta=0$.

Market selection is less likely to favor a unique agent because a position in the second asset is a safe bet. In Fig. 5, we plot the areas of dominance and survival for all the possible combinations of beliefs of agent 1 and parameter $r=g_{d} / g_{u}$ when $\delta=0.5$ and $\pi^{2}=(0.6,0.4)$.

Trinomial Tree We continue our examples by exploring the outcomes of market selection under complete and incomplete markets. First, we consider the market structure with two assets and three states of the world shown in Sect. 2.1 with $r_{u}=r_{m}=0.2$. We also choose $\pi=(1 / 3,1 / 3,1 / 3), \delta=0.5, \pi^{1}=\left(3 \pi_{1,2}^{1} / 4, \pi_{1,2}^{1} / 4,1-\pi_{1,2}^{1}\right)$, and $\pi^{2}=\left(\pi_{1,2}^{2} / 4,3 \pi_{1,2}^{2} / 4,1-\pi_{1,2}^{2}\right)$, for two given parameters $\pi_{1,2}^{1}$ and $\pi_{1,2}^{2}$.

In the upper plot of Fig. 6 , the areas of dominance and survival are similar to those in the first plot of Fig. 4, the only difference is that now the "truth" corresponds 

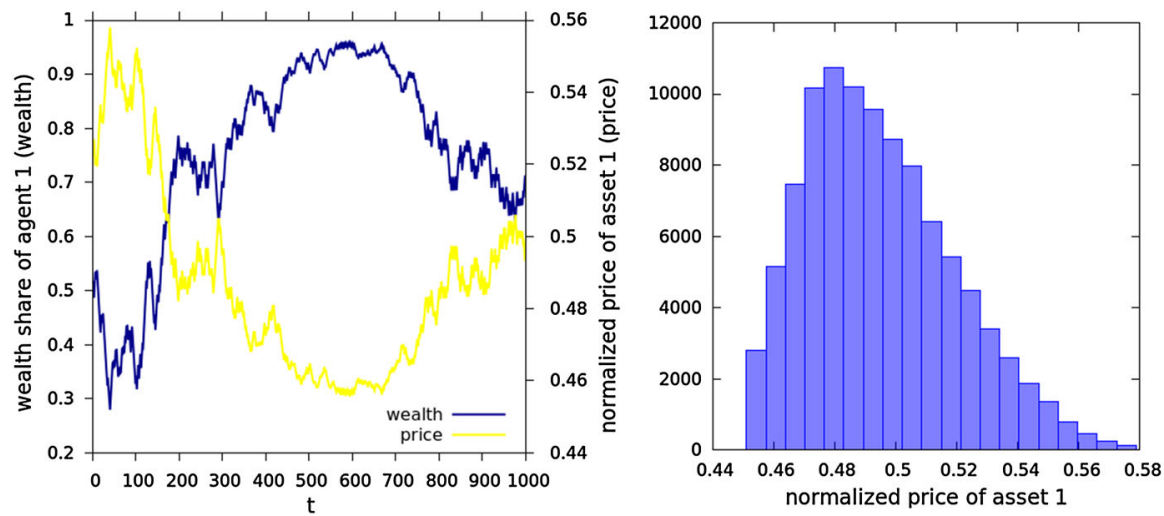

Fig. 3 Left panel: dynamics of agent 1's relative wealth share and the normalized price of asset 1 in an economy with two diagonal assets and $\delta=0.8, \pi=(0.5,0.5), \pi^{1}=(0.45,0.55), \pi^{2}=(0.6,0.4)$, and $w_{0}=0,5$. Both agents survive. Right panel: histogram of asset 1's normalized price over 100,000 periods under the same settings of the first panel

Fig. 4 Areas of dominance and survival in a binomial tree economy as a function of $\pi^{1}$ and $\pi^{2}$ (upper panel) and $\pi^{1}$ and $\delta$ (lower panel). 1D: agent 1 dominates; $2 D$ : agent 2 dominates; $H$ : long-run heterogeneity; $r=g_{d} / g_{u}=0.2$ (color figure online)
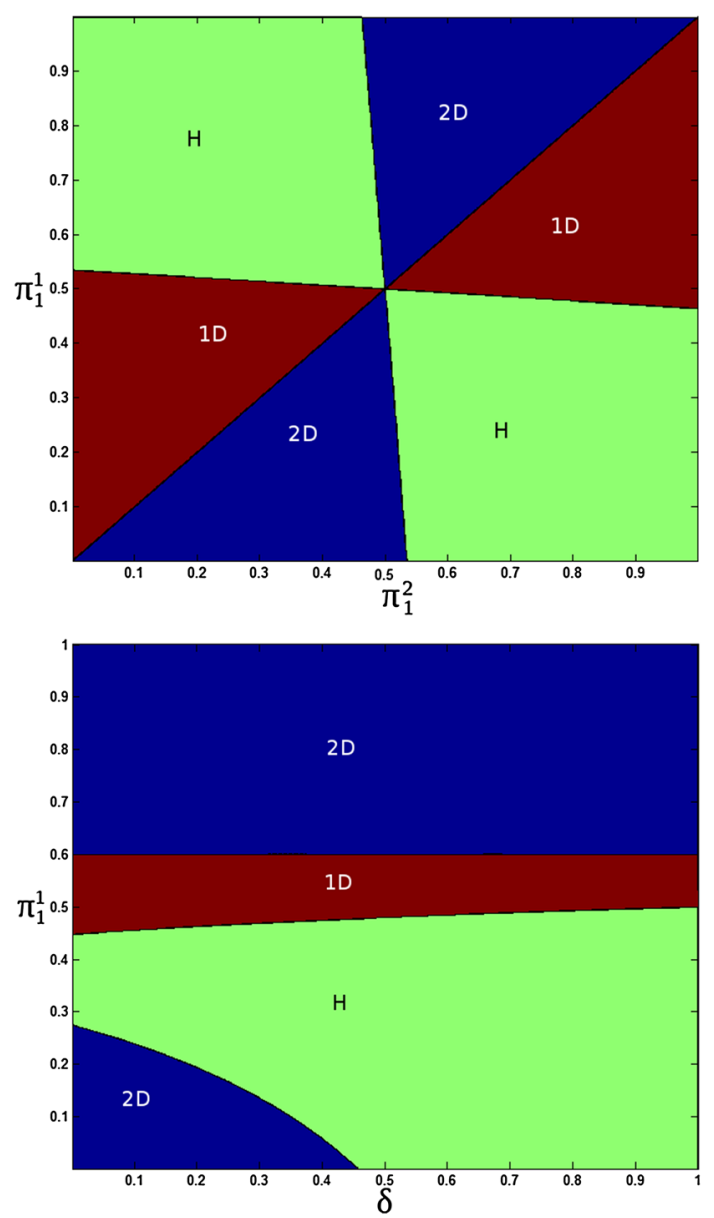
Fig. 5 Areas of dominance and survival in a binomial tree economy as a function of $r$ and $\pi^{1} . \delta=0.5$ and $\pi^{2}=$ $(0.6,0.4)$. 1D: agent 1 dominates; $2 D$ : agent 2 dominates; $H$ : long-run eterogeneity (color figure online)

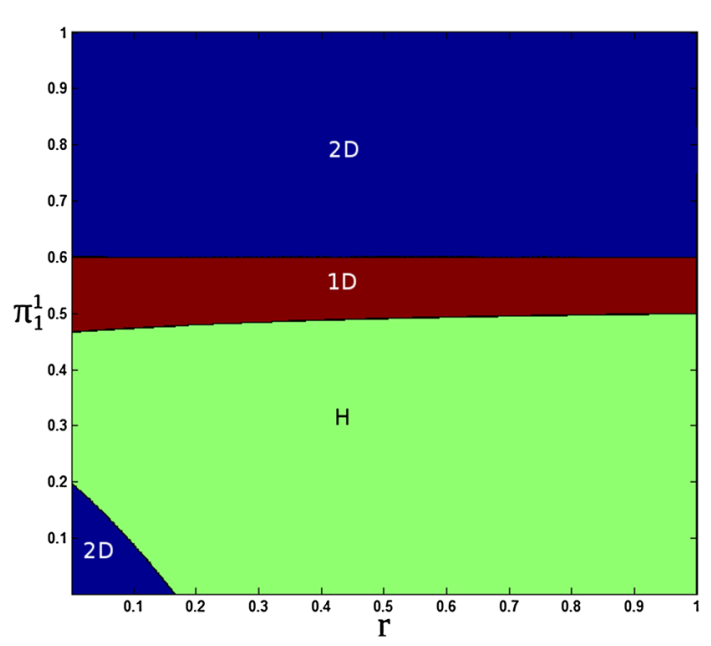

to the sum of probabilities of the first two states, hence $2 / 3$. In the lower plot, the same analysis is performed with complete markets, leading to a much larger area of long-run heterogeneity. Completing the market offers a way for agents with anticorrelated beliefs about the probability of states 1 and 2 to take different positions in these two assets, positions that with incomplete markets cannot be taken. As a consequence, the possible combinations of beliefs that produce long-run heterogeneity increase.

Obviously, the role played by the belief structure is fundamental. To see it, consider a slightly different belief structure for agent $1: \pi^{1}=\left(\pi_{1,2}^{1} / 2, \pi_{1,2}^{1} / 2,1-\right.$ $\pi_{1,2}^{1}$ ). In this situation, agent 1 should be favored since she can distribute more evenly (hence in accordance with the underlying stochastic process) her wealth among assets. Indeed, when $\pi_{1,2}^{1}=2 / 3$, she plays the Generalized Kelly rule $x^{*}$; hence, she dominates no matter the value of $\pi_{1,2}^{2}$. Figure 7 confirms the intuition: The area where agent 1 dominates increases and occupies a large portion of the plot.

This exercise provides a link with the work of Fedyk et al. (2013) about the welfare effect of enlarging the asset span. Considering a general equilibrium model where one agent has correct beliefs and one has incorrect beliefs, the authors show that the possibility of trading several risky assets does not increase welfare in general while, in most of the cases, it causes a severe welfare loss. A basic feature of their model is that the agent with correct beliefs dominates no matter how many assets are traded; thus, the divergence in terms of welfare is triggered by the speed at which the inaccurate agent loses everything. Figures 6 and 7 show, instead, how in our model there exists combinations of beliefs such that, when the asset span increases, the dominant agent changes from agent 2 to agent 1 or we pass from the dominance of one of the two agents to the survival of both. Hence, establishing whether a larger asset span can cause a welfare loss becomes much more 
Fig. 6 Areas of dominance and survival in a trinomial tree economy as a function of agents' beliefs. Upper panel: incomplete markets. Lower panel: complete markets. $1 D$ : agent 1 dominates; $2 D$ : agent 2 dominates; $H$ : long-run heterogeneity (color figure online)

Fig. 7 Areas of dominance and survival in a trinomial tree economy as a function of agents' beliefs when markets are complete. $1 D$ : agent 1 dominates; $2 D$ : agent 2 dominates; $H$ : long-run heterogeneity (color figure online)
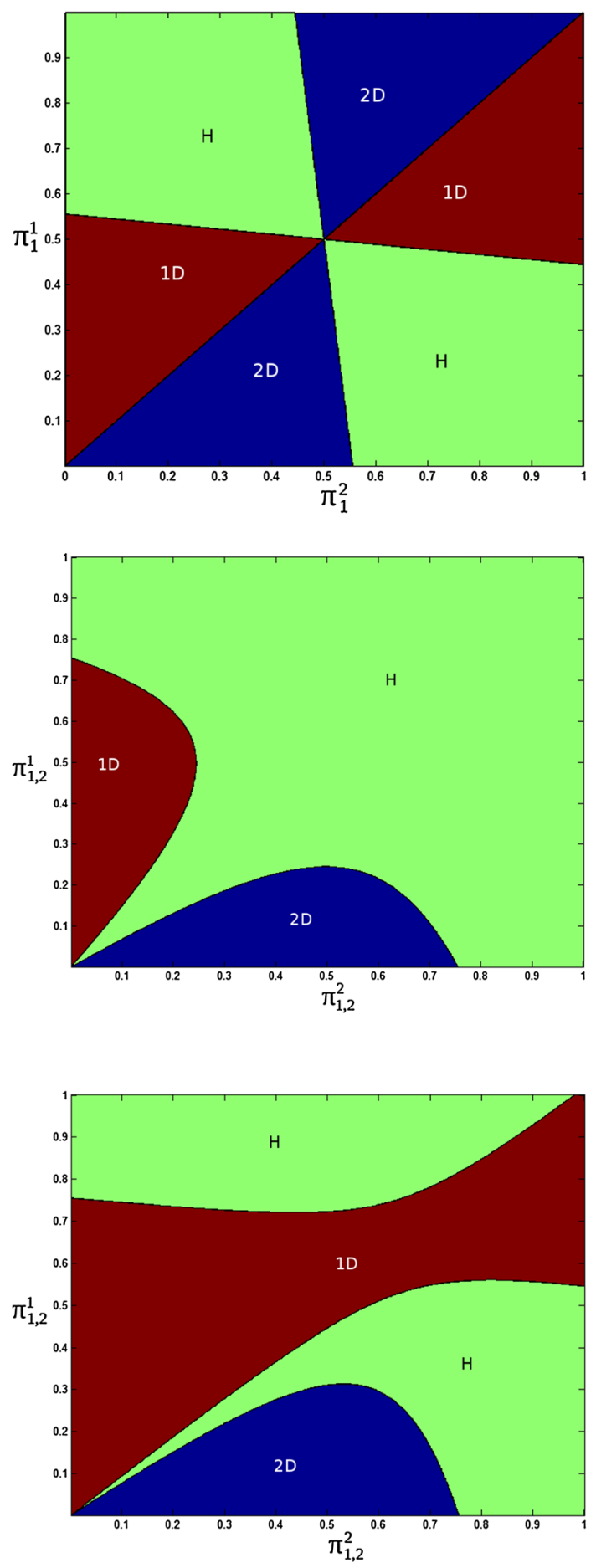
Fig. 8 Areas of dominance and survival in a three-agent economy with $D=\mathbb{I}$. $1 S$ : agent 1 survives; $1 D$ : agent 1 dominates; $1 \mathrm{~V}$ : agent 1 vanishes; 2, 3S: both agent 2 and 3 survive; $H$ : at least two agents survive; 1, 2, 3S: all three agents survive; ?: unknown (color figure online)

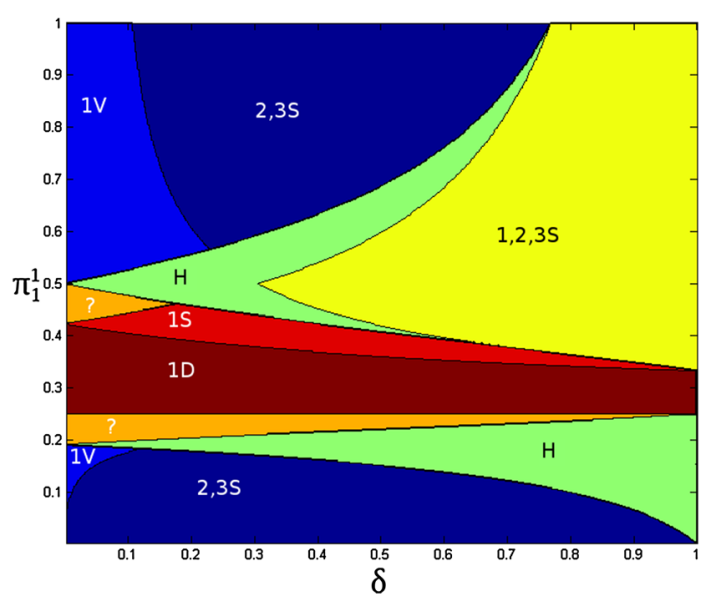

complex. Indeed, it could be the case that enlarging the asset span increases total welfare. ${ }^{16}$

\subsection{Three-agent economy}

In this section, we investigate market selection outcomes in an economy with three states of the world, complete markets, and three Subjective Generalized Kelly agents. Assume $D=\mathbb{I}$ and $\pi=(1 / 3,1 / 3,1 / 3)$. We fix $\pi^{1}=\left(\pi_{1}^{1},\left(1-\pi_{1}^{1}\right) / 2,\left(1-\pi_{1}^{1}\right) / 2\right)$, $\pi^{2}=(1 / 4,1 / 2,1 / 4)$ and $\pi^{3}=(1 / 4,1 / 4,1 / 2)$. Figure 8 shows the possible long-run outcomes as a function of $\delta$ and $\pi_{1}^{1}$.

Differently from two-agent economies, in a three-agent economy our sufficient conditions are not tight. Thus, there exist combinations of $\pi_{1}^{1}$ and $\delta$ for which we cannot characterize long-run outcomes. Consider the regions $1 \mathrm{~S}$ and $1 \mathrm{D}$ first. For these combinations of $\pi_{1}^{1}$ and $\delta$ agent 1 survives. Indeed, choosing the group $I=\{1\}$ we have $\mu^{I}(0)>0$. In the region $1 \mathrm{D}$, around the truth, we also have $\underline{\mu}^{I}(1)>0$ so that agent 1 dominates. In the areas $1 \mathrm{~V}$ and $2,3 \mathrm{~S}, \bar{\mu}^{I}(0)<0$ and $\bar{\mu}^{I}(1)<0$ : Agent 1 vanishes and group $I^{c}=\{2,3\}$ dominates. Regarding the fate of agents 2 and 3 , both can survive or one of the two dominates. Define $I^{\prime}=\{2\}$ and $I^{\prime \prime}=\{3\}$, in the regions $2,3 \mathrm{~S} \mu^{I^{\prime}}(0)>0$ and $\mu^{I^{\prime \prime}}(0)>0$, so that we are sure that both agents survive.

Continuing the analysis, in the region $1,2,3 \mathrm{~S} \mu^{I}(0)>0, \mu^{I^{\prime}}(0)>0$, and $\mu^{I^{\prime \prime}}(0)>$ 0 , hence all agents survive. In the region $\mathrm{H}, \bar{\mu}^{I}(1)<0, \bar{\mu}^{I^{\prime}}(1)<0$, and $\bar{\mu}^{I^{\prime \prime}}(1)<0$, so that no one dominates. This is equivalent to say that at least two agents survive. Finally, there also exists a region, marked with ?, where our sufficient conditions are too weak to characterize the market selection outcome.

Thus, also in an asset economy with three agents there exists a region where multiple agents survive. Set, for example, $\pi^{1}=(0.6,0.2,0.2), \delta=0.8$, and $w_{0}^{1}=w_{0}^{2}=w_{0}^{3}=$

\footnotetext{
16 It remains the difficulty to measure welfare in a framework such ours where rules are not explicitly derived from an utility maximization.
} 


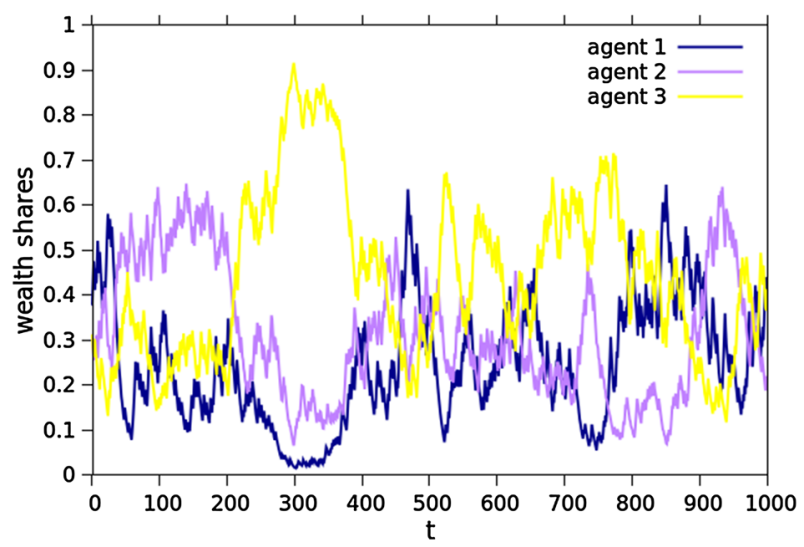

Fig. 9 Dynamics of agents' relative wealth shares in an economy with three diagonal assets and three agents. $\pi^{1}=(0.6,0.2,0.2), \pi^{2}=(1 / 4,1 / 2,1 / 4), \pi^{3}=(1 / 4,1 / 4,1 / 2)$, and $\delta=0.8$, and $w_{0}^{1}=w_{0}^{2}=$ $w_{0}^{3}=1 / 3$. All three agents survive

$1 / 3$, then all agents survive. Figure 9 shows a realization of agents' relative wealth. Around period $t=300$, and again at $t \approx 750$, agent 1 has lost almost all her wealth. However, in later periods, she recovers. Notice also that agents' relative wealth does not display any stable ordering.

\subsection{Fixed-mix rules derived under correct beliefs and different limit risk preferences}

Throughout the paper, we have assumed that agents employ Subjective Generalized Kelly rules, $\mathbf{R} 1-\mathbf{R} 2$. Given that this rule is log-optimal in the limit of the agent using it having all the wealth and that market selection favors log-optimal portfolios, it is not entirely surprising that if there is an agent who knows the truth, then she dominates, see also Corollaries 2-3.

In this section, we take a different approach and use fixed-mix rules to model agents who have correct beliefs about the asset dividend process but have different limit risk preferences. ${ }^{17}$ In parallel to the log-optimality of the Subjective Generalized Kelly rule when its wealth share is unitary, we shall derive each agents' fixed-mix rule as a rule that is optimal for an agent with recursive utility of the Epstein-Zin type with unitary intertemporal elasticity of substitution (IES) coefficient, discount factor equal to $\delta$, and coefficient of relative risk aversion $\gamma$, in the partial equilibrium limit of the agent using such rule being alone in the economy. ${ }^{18}$ Following Epstein and Zin (1989) and imposing unitary IES parameter, agent $i$ with correct beliefs $\pi$, discount factor $\delta$, and relative risk aversion $\gamma^{i}$ maximizes a recursive utility of the type

\footnotetext{
17 This exercise is in the spirit of Sciubba (2006) where a CAPM-based heuristic rule, a log-optimal rule, and a mean-variance rule, all derived under the truth, are compared.

18 Dindo (2015) deals with such rules in a general equilibrium model.
} 
Fig. 10 Areas of dominance and survival in a market with $N=2$ agents and "quasi-optimal" rules derived from different relative risk aversion coefficients $\gamma^{1}$ and $\gamma^{2}$. The aggregate endowment is risky and $g_{d}=0.2 g_{u}$. Long-lived assets are such that $D=\mathbb{I}$. $1 D$ : agent 1 dominates. $2 D$ : agent 2 dominates. $H$ : long-run heterogeneity (color figure online)

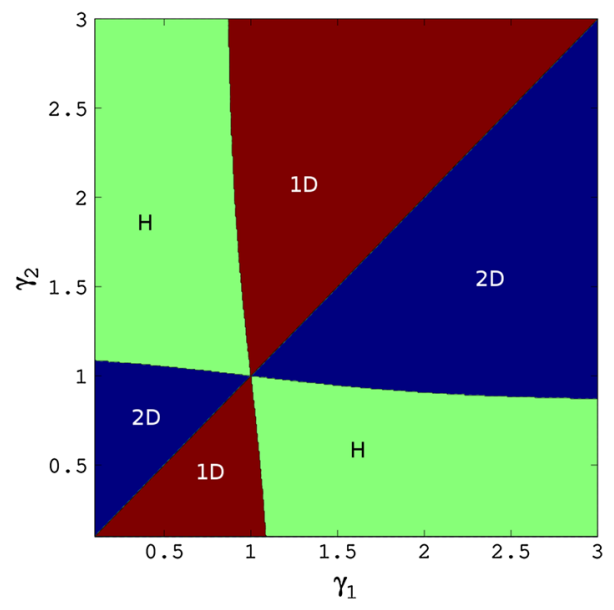

$$
U_{t}^{i}=C_{t}^{1-\delta}\left(\mathrm{E}^{\pi}\left[\left(U_{t+1}^{i}\right)^{1-\gamma^{i}}\right]\right)^{\frac{\delta}{1-\gamma^{i}}}, t \in \mathbb{N}_{0}
$$

under the sequential budget constraint (1) and where asset prices are derived by imposing that she is the representative agent. The unitary IES coefficient and the homogeneous discount factor $\delta$ imply that all agents save at the same rate $\delta$, so that $\mathbf{R 3}$ holds. $\mathbf{R} 1$ is assumed, so that the portfolio rule is kept fixed also when the wealth distribution, and thus also equilibrium prices, change. Provided R2, it can be shown that for all $\gamma^{i} \in(0,+\infty)$ the vector of portfolio rules is in the interior of the cone generated by the $S$ columns of the matrix $D$ (a sufficient conditions to avoid arbitrages in equilibrium, see the discussion after Proposition 1 and its proof for more details). We can thus still employ Propositions 2-3 to determine long-run outcomes.

Figure 10 shows the long-run outcomes in a two-agent economy with diagonal long-lived assets when the aggregate endowment has two possible growth rates: $g_{u}$ in state $s=1$ and $g_{d}$ in state $s=2$. The truth is $\pi=(0.5,0.5)$. Imposing optimality in the limit of having all the wealth, agent- $i$ portfolio rule is

$$
x_{1}^{i}=\frac{\pi_{1} g_{u}^{1-\gamma^{i}}}{\pi_{1} g_{u}^{1-\gamma^{i}}+\pi_{2} g_{d}^{1-\gamma^{i}}} \quad \text { and } \quad x_{2}^{i}=\frac{\pi_{2} g_{d}^{1-\gamma^{i}}}{\pi_{1} g_{u}^{1-\gamma^{i}}+\pi_{2} g_{d}^{1-\gamma^{i}}} \text {. }
$$

The fact that there is aggregate risk is crucial in making the resulting quasi-optimal fixed rule depending on the risk aversion coefficient $\gamma .{ }^{19}$ The $\gamma=1$ case is still a benchmark and corresponds to the portfolio $x^{*}=\pi$, the Generalized Kelly rule. Higher (lower) relative risk aversion implies a smaller (larger) position in the asset that pays the dividend when the high (low) growth rate is realized. The figure shows that long-run heterogeneity still occurs provided agents' risk aversions are on opposite

\footnotetext{
19 Under no aggregate risk, fair pricing holds for each representative agent economy. As a result, all rules coincide when beliefs are homogeneous.
} 
sides with respect to the $\gamma=1$ benchmark. Keeping an agent risk aversion fixed, say the one of agent 2, the closer agent 1 agent is to the $\gamma=1$ benchmark, the more likely she dominates. The intuition is similar to the one developed for Subjective Generalized Kelly rules as also heterogeneity of risk preferences can be mapped into effective beliefs. Having $\gamma>1(\gamma<1)$ corresponds to pessimism (optimism) in the limit of having all the wealth. Effective beliefs may become closer or further away from the truth depending on the risk aversion of the other agent when the latter has most of the wealth.

\section{Conclusion}

In this paper, we investigate the MSH in an exchange economy with long-lived assets where agents have homogeneous saving rates, heterogeneous beliefs, and employ Subjective Generalized Kelly rules, a particular type of fixed-mix portfolios. In this framework, Evstigneev et al. (2008) prove that if there exists an agent with correct beliefs, then she gains all the wealth in the long-run. Asset prices converge to those of a Lucas' model where the representative agent has logarithmic instantaneous utility. We instead focus on an economy where agents have heterogeneous beliefs and provide sufficient conditions for an agent to have a positive, null, or unitary fraction of wealth share in the long-run.

Our main finding is that there exist distributions of agents' beliefs such that agents' heterogeneity is the long-run outcome. Moreover, this result is generic and robust to local perturbation of beliefs. We show that our results are due to the non-optimality of fixed-mix rules in the limit of an agent having a negligible share of the total wealth. Non-accuracy of beliefs and non-optimality of the rules balance each other and lead to survival instead of vanishing.

\section{A Proofs of Theorems and Lemmas}

\section{A.1 Proof of Lemma 1}

Let $\bar{\alpha}=\max _{i \in N, k \in K}\left\{\alpha_{k}^{i}\right\}$ and $\bar{\delta}=\max _{i \in N}\left\{\sum_{k=1}^{K} \alpha_{k}^{i}\right\}$. Consider the matrix $A(W ; \alpha)$ in (9) whose element $A_{k, l}$ reads

$$
A_{k, l}=\frac{\sum_{i=1}^{N} \alpha_{k}^{i} \alpha_{l}^{i} W^{i}}{\sum_{j=1}^{N} \alpha_{l}^{j} W^{j}}
$$

From R1 and R2, it follows that $0<A_{k, l}<\bar{\alpha}<1$ for all $k, l$, and that $0<$ $\sum_{k=1}^{K} A_{k, l}<\bar{\delta}<1$ for all $l$. The first group of inequalities implies

$$
|\mathbb{I}-A|_{l, l}-\sum_{k=1, k \neq l}^{K}|\mathbb{I}-A|_{k, l}=\sum_{k=1}^{K}(\mathbb{I}-A)_{k, l}
$$


while the second group implies $\sum_{k=1}^{K}(\mathbb{I}-A)_{k, l}=1-\sum_{k=1}^{K} A_{k, l}>1-\bar{\delta}>0$. Thus, the matrix $\mathbb{I}-A$ is column strictly diagonally dominant and, by the Levy-Desplanques theorem (Taussky 1949), invertible.

\section{A.2 Proof of Proposition 1}

The first part of the statement follows from Lemma 1 and from the derivation in the text before the proposition.

Regarding the absence of arbitrages consider the following. According to Stiemke's Lemma, the absence of arbitrage is equivalent to the existence of a vector $q \in \mathbb{R}_{++}^{S}$ such that $R(W ; \alpha, D) q=P$ or, with (12),

$$
D q=(\mathbb{I}-A(W ; \alpha)) P=\left[\begin{array}{ccc}
1-\frac{\sum_{i=1}^{N} \alpha_{1}^{i} \alpha_{1}^{i} W^{i}}{\sum_{j=1}^{N} \alpha_{1}^{j} W^{j}} & \cdots & -\frac{\sum_{i=1}^{N} \alpha_{1}^{i} \alpha_{K}^{i} W^{i}}{\sum_{j=1}^{N} \alpha_{K}^{j} W^{j}} \\
\vdots & \ddots & \vdots \\
-\frac{\sum_{i=1}^{N} \alpha_{K}^{i} \alpha_{1}^{i} W^{i}}{\sum_{j=1}^{N} \alpha_{1}^{j} W^{j}} & & 1-\frac{\sum_{i=1}^{N} \alpha_{K}^{i} \alpha_{K}^{i} W^{i}}{\sum_{j=1}^{N} \alpha_{K}^{j} W^{j}}
\end{array}\right]\left[\begin{array}{c}
\sum_{j=1}^{N} \alpha_{1}^{j} W^{j} \\
\vdots \\
\vdots \\
\sum_{j=1}^{N} \alpha_{K}^{j} W^{j}
\end{array}\right]
$$

Thus, the $k$ th component of $(\mathbb{I}-A(W ; \alpha)) P$ reads $\sum_{i=1}^{N}\left[\left(1-\delta^{i}\right) \delta^{i} W^{i}\right] x_{k}^{i}$. By $\mathbf{R} 1$ it is $x_{k}^{i}=\sum_{s=1}^{S} \pi_{s}^{i} d_{k}(s)$; hence, one has

$$
D q=D\left[\begin{array}{c}
\sum_{i=1}^{N}\left[\left(1-\delta^{i}\right) \delta^{i} W^{i}\right] \pi_{1}^{i} \\
\vdots \\
\sum_{i=1}^{N}\left[\left(1-\delta^{i}\right) \delta^{i} W^{i}\right] \pi_{S}^{i}
\end{array}\right]
$$

That is, calling $q_{s}$ the $s$ th component of $q$ and given that $D$ has full rank by D4, it is $q_{s}=\sum_{i=1}^{N}\left[\left(1-\delta^{i}\right) \delta^{i} W^{i}\right] \pi_{s}^{i}$. R1, R2, and the first part of the Proposition imply $q_{s}>0 \forall s \in S$ so that the statement is proven.

\section{A.3 Proof of Propositions 2}

As we shall show, the stochastic process that corresponds to the two groups' relative wealth dynamics has bounded increments. As a result, we can prove the proposition by applying Theorem 2.1 in Bottazzi and Dindo (2015).

Consider the variable

$$
z_{t}^{I}=\log \frac{w_{t}^{I}}{1-w_{t}^{I}}
$$


such that $z_{t}^{I}=z_{t-1}^{I}+g^{I}\left(\sigma_{t}\right)$, with $g^{I}\left(\sigma_{t}\right)=\log G^{I}\left(\sigma_{t}\right)$ and

$$
G^{I}\left(\sigma_{t}\right)=\frac{\sum_{k=1}^{K} r_{k, s_{t}}\left(w_{t-1} ; x, \delta, D\right) \beta_{k}^{I}\left(w_{t-1} ; x\right)}{\sum_{k=1}^{K} r_{k, s_{t}}\left(w_{t-1} ; x, \delta, D\right) \beta_{k}^{I^{c}}\left(w_{t-1} ; x\right)} .
$$

One has the following.

Lemma 5 The process $z_{t}^{I}$ has bounded increments, that is, there exists a $B \in \mathbb{R}$ such that $\left|z_{t}^{I}-z_{t-1}^{I}\right|<B$ P-a.s..

Proof By $\mathbf{R 3}$ there exists a small enough $\varepsilon>0$ such that $\varepsilon \leq x_{k}^{i} \leq 1-\varepsilon \forall i, k$. Since

$$
\beta_{k}^{I}\left(w_{t} ; x\right)=\frac{x_{k}^{I}\left(w_{t} ; x\right)}{\sum_{i=1}^{N} x_{k}^{i} w_{t}^{i}}
$$

for any asset $k$ and any time $t$ it is

$$
\frac{\varepsilon}{1-\varepsilon} \leq \beta_{k}^{I}\left(w_{t} ; x\right) \leq \frac{1-\varepsilon}{\varepsilon}
$$

Consider the matrix

$$
\begin{aligned}
r_{k, s}\left(w_{t} ; x, \delta, D\right) & =\left[(1-\delta)\left(\mathbb{I}-\delta A\left(w_{t} ; x\right)\right)^{-1} D\right]_{k, s} \\
& =(1-\delta) d_{k, s}+\delta p_{k, s}\left(w_{t} ; x, \delta, D\right)
\end{aligned}
$$

It holds: $0<r_{k, s}\left(w_{t} ; x, \delta, D\right)<1 \forall k, t, s$ and $\sum_{k=1}^{K} r_{k, s}\left(w_{t} ; x, \delta, D\right)=1 \forall s, t$. Thus for any group $I$, state $s$ and time $t$ one has

$$
\frac{\varepsilon}{1-\varepsilon} \leq \sum_{k=1}^{K} r_{k, s}\left(w_{t} ; x, \delta, D\right) \beta_{k}^{I}\left(w_{t} ; x\right) \leq \frac{1-\varepsilon}{\varepsilon} .
$$

Given (25) and (26), by direct algebraic substitution it is straightforward to verify that

$$
2 \log \frac{\varepsilon}{1-\varepsilon} \leq z_{t}^{I}-z_{t-1}^{I} \leq 2 \log \frac{1-\varepsilon}{\varepsilon}
$$

and the statement is proven.

In order to prove Proposition 2, use the notation of Bottazzi and Dindo (2015) and call $\mu_{t}\left(z^{I}\right)$ the date $t$ conditional drift of the process $\left\{z_{t}^{I}\right\}, \mu_{t}\left(z^{I}\right)=\mathrm{E}^{\mathrm{P}}\left[z_{t+1}^{I}-\right.$ $\left.z_{t}^{I} \mid \Im_{t}, z_{t}^{I}=z^{I}\right]$. If $\mu^{I}(w ; v)$ is an element of the set $\left\{\mu^{I}(w) \mid w \in \Delta^{N}, w^{I}=v\right\}$, with $\mu^{I}(w)$ defined in (19), then by construction

$$
\mu_{t}\left(z^{I}\right)=\mu^{I}\left(w_{t} ; v=\frac{e^{z^{I}}}{1+e^{z^{I}}}\right) .
$$


Having fixed $z^{I}$, and thus $v$, the precise value of $w_{t}$ still depends on the specific element of $\mathfrak{\Im}_{t}$ considered.

We shall start from the proof of statement (i). If $\mu^{I}(0)>0$, then, given the property of the lower bound and the continuity of $\mu^{I}(w ; v)$ with respect to $v$, there exist $\varepsilon>0$ and $M>0$ such that, for all $z^{I}<-M$ and $t$, it is $\mu_{t}\left(z^{I}\right)>\varepsilon$. Since $z_{t}^{I}$ has bounded increments, Theorem 2.1 in Bottazzi and Dindo (2015) applies and Prob $\left\{\lim \sup _{t \rightarrow \infty} z_{t}^{I}>-\infty\right\}=1$. Provided $\bar{\mu}^{I}(1)>0$, the same reasoning applies to prove (ii), see also Corollary 2.1 of Bottazzi and Dindo (2015).

\section{A.4 Proof of Lemma 2}

Let us consider the process $z_{t}^{I}$ in (25) and all the other quantities defined at the beginning of appendix A.3. We begin with the following Lemma.

Lemma 6 If the set of rules are not overlapping, $\mathbf{R 4}$, and if there are no redundant assets, D4, then $z_{t}^{I}$ does not possess any deterministic fixed point, that is, \# z s.t. $\mathrm{P}\left(z_{t}^{I}=z \mid z_{t^{\prime}}^{I}=z\right)=1 \forall t>t^{\prime}$.

Proof Suppose such $z$ exists and at a certain time $t-1$ it is $z_{t-1}^{I}=z$. Then, by definition, it holds that $z_{t}^{I}-z_{t-1}^{I}=0$ for all the possible states of the world $s=$ $1,2, \ldots, S$, so that

$$
\sum_{k=1}^{K} r_{k, s}\left(w_{t-1} ; x, \delta, D\right)\left(\beta_{k}^{I}\left(w_{t-1} ; x\right)-\beta_{k}^{I^{c}}\left(w_{t-1} ; x\right)\right)=0 \quad \forall s=1,2, \ldots, S
$$

That is

$$
\left(\beta^{I}\left(w_{t-1} ; x\right)-\beta^{I^{c}}\left(w_{t-1} ; x\right)\right)\left(\left(\mathbb{I}-\delta A\left(w_{t-1} ; x\right)\right)^{-1} D\right)=\mathbf{0}
$$

The trivial solution $\beta^{I}=\beta^{I^{c}}$ is excluded by $\mathbf{R 4}$, and according to Proposition 1 , the kernel of $\left(\mathbb{I}-\delta A\left(w_{t-1}, x\right)\right)^{-1} D$ is zero, implying that the system of equations has no solution and the statement is proven.

The proof proceeds by noticing that $G^{I}$ in (26) depends on history $\sigma_{t}$ through the wealth distribution $w_{t}$ and the last realized state $s_{t}$. Given the distribution $w \in \Delta^{N}$ define

$$
\bar{G}^{I}\left(\sigma_{t}\right)=\max _{s=1, \ldots, S}\left\{\left|G^{I}\left(w, s_{t}\right)\right|\right\}
$$

which, being the upper envelope of continuous functions, is a continuous function on the compact set $\Delta^{N}$. Then, by the Weierstrass theorem, it has a minimum $\underline{G}$. Moreover, it is $\underline{G}>0$ because, otherwise, $z_{t}^{I}$ would have a deterministic fixed point, which is not possible by Lemma 6. Then 


$$
\operatorname{Prob}\left\{\left|z_{t}^{i}-z_{t-1}^{i}\right| \geq \underline{g} \mid \Im_{t-1}\right\} \geq \rho=\min \left\{\pi_{1}, \ldots, \pi_{S}\right\} .
$$

and by taking $\gamma=\min \{\underline{g}, \rho\} / 2$ the assertion is proven.

\section{A.5 Proof of Lemma 3}

Let us consider the first statement. If it is wrong, then

$$
\frac{w_{t+1}^{I^{c}}}{w_{t}^{I^{c}}}-\frac{w_{t+1}^{I}}{w_{t}^{I}}=\sum_{k=1}^{K}\left(\beta_{k}^{I^{c}}\left(w_{t} ; x\right)-\beta_{k}^{I}\left(w_{t} ; x\right)\right) r_{k, s}\left(w_{t} ; x, \delta, D\right) \geq 0 \quad \forall s
$$

and, since the process does not admit any deterministic fixed point (c.f. Lemma 6), the inequality is strict for some $s^{\prime}$. By construction it is

$$
\sum_{k=1}^{K}\left(\beta_{k}^{I^{c}}\left(w_{t} ; x\right)-\beta_{k}^{I}\left(w_{t} ; x\right)\right) p_{k, t}=0
$$

Together with (27) the latter implies that $\beta^{I^{c}}\left(w_{t} ; x\right)-\beta^{I}\left(w_{t} ; x\right)$ would be a weak arbitrage, which contradicts the hypotheses. For the second statement one can reason following the same lines and, in order to complete the proof, it is enough to choose $\epsilon=\min _{s}\left\{\pi_{s}\right\} / 2$.

\section{A.6 Proof of Proposition 3}

Consider the process $z_{t}^{I}$ in (25) and all the other quantities defined at the beginning of appendix A.3. Then we can directly apply Lemma 5 and $z_{t}^{I}$ has bounded increments. $\mathbf{R} 4$ and the lack of arbitrages imply by Lemma 3

$$
\begin{aligned}
\operatorname{Prob}\left\{z_{t+1}^{I}-z_{t}^{I}>0 \mid \Im_{t}\right\} & =\operatorname{Prob}\left\{\frac{w_{t+1}^{I}}{w_{t+1}^{I^{c}}}>\frac{w_{t}^{I}}{w_{t}^{I^{c}}} \mid \Im_{t}\right\} \\
& =\operatorname{Prob}\left\{\frac{w_{t+1}^{I}}{w_{t}^{I}}>\frac{w_{t+1}^{I^{c}}}{w_{t}^{I^{c}}} \mid \Im_{t}\right\}>\epsilon .
\end{aligned}
$$

Moreover, invoking Lemma 2, one has

$$
\operatorname{Prob}\left\{\left|z_{t+1}^{I}-z_{t}^{I}\right|>\gamma \mid \mathfrak{\Im}_{t}\right\}=\operatorname{Prob}\left\{\left|\log \frac{w_{t+1}^{I}}{w_{t+1}^{I^{c}}}-\log \frac{w_{t}^{I}}{w_{t}^{I^{c}}}\right|>\gamma \mid \mathfrak{s}_{t}\right\}>\gamma
$$

Thus, defining $\epsilon_{L}=\min \{\epsilon, \gamma\} / 2$, one gets $\operatorname{Prob}\left\{z_{t+1}^{I}>z_{t}^{I}+\epsilon_{L} \mid \mathfrak{\Im}_{t}\right\}>\epsilon_{L}$, meaning that the process $z_{t}^{I}$ has finite positive increments. A symmetric argument shows that $z_{t}^{I}$ has also negative finite increments. 
We shall start from the proof of statement (i) and (ii). Using the notation of Bottazzi and Dindo (2015), we call $\mu_{t}\left(z^{I}\right)$ the date $t$ conditional drift of $\left\{z_{t}^{I}\right\}$, $\mu_{t}\left(z^{I}\right)=\mathrm{E}^{\mathrm{P}}\left[z_{t+1}^{I}-z_{t}^{I} \mid \mathfrak{\Im}_{t}, z_{t}^{I}=z^{I}\right]$. If $\mu^{I}(w ; v)$ is an element of the set $\left\{\mu^{I}(w) \mid w \in\right.$ $\left.\Delta^{N}, w^{I}=v\right\}$, with $\mu^{I}(w)$ defined in (19), then by construction

$$
\mu_{t}\left(z^{I}\right)=\mu^{I}\left(w_{t} ; v=\frac{e^{z^{I}}}{1+e^{z^{I}}}\right) .
$$

Having fixed $z^{I}$, and thus $v$, the precise value of $w_{t}$ still depends on the specific element of $\mathfrak{s}_{t}$ considered.

By continuity of $\mu^{I}(w ; v)$ with respect to $v$ and by the definition of lower bound, the conditions $\underline{\mu}^{I}(0)>0$ and $\underline{\mu}^{I}(1)>0$ imply that there exist $\varepsilon>0$ and $M>0$ such that, for any $t$, it is $\mu_{t}\left(z^{\bar{I}}\right)>\varepsilon$ if $z^{I}>M$ or $z^{I}<-M$. Using Theorem 3.1 from Bottazzi and Dindo (2015), it follows that Prob $\left\{\lim _{t \rightarrow \infty} z_{t}^{I}=+\infty\right\}=1$, group $I$ dominates. Conversely, conditions $\bar{\mu}^{I}(0)<0$ and $\bar{\mu}^{I}(1)<0$ imply that there exist $\varepsilon>0$ and $M>0$ such that, for any $t$, it is $\mu_{t}\left(z^{I}\right)<-\varepsilon$ if $z^{I}>M$ or $z^{I}<-M$. Using Corollary 3.1 of Bottazzi and Dindo (2015), it follows that Prob $\left\{\lim _{t \rightarrow \infty} z_{t}^{I}=-\infty\right\}=1$ and group $I$ vanishes.

In order to prove statement (iii) note that, by Proposition 2, we already know that both groups survive. Thus, we need to prove that for $G=I, I^{c}$

$$
\operatorname{Prob}\left\{\liminf _{t \rightarrow \infty} w_{t}^{G}=0 \text { and } \limsup _{t \rightarrow \infty} w_{t}^{G}=1\right\}=1 .
$$

Assume by contradiction that for a group $G$

$$
\operatorname{Prob}\left\{\liminf _{t \rightarrow \infty} w_{t}^{G}=0 \text { and } \limsup _{t \rightarrow \infty} w_{t}^{G}=1\right\}<1 .
$$

Then, there must exist a positive measure set $\Sigma^{\prime} \subset \Sigma$ such that for all $\sigma \in \Sigma^{\prime}$

$$
\liminf _{t \rightarrow \infty} w_{t}^{G}(\sigma)>0 \text { or } \limsup _{t \rightarrow \infty} w_{t}^{G}(\sigma)<1 .
$$

The latter is in contradiction with the process $\left\{z_{t}^{I}\right\}$ having finite positive and negative increments.

\section{A.7 Proof of Corollary 2}

Consider the group formed by agent $i$ alone, where $\pi^{i}=\pi$, and call $\mu^{i}(w ; v)$ a generic element of the set $\left\{\mu^{i}(w) \mid w \in \Delta^{N}, w^{i}=v\right\}$. When $v=1$ agent $i$ owns all the wealth and normalized prices correspond to her portfolio rule. Thus, from (19) one gets

$$
\mu^{i}(w ; 1)=-\sum_{s=1}^{S} \pi_{s} \log \left(\delta+(1-\delta) \sum_{k=1}^{K} d_{k, s} \frac{x_{k}^{i^{c}}(w, x)}{x_{k}^{i}}\right)
$$


where $x^{i^{c}}(w, x)$ is the aggregate portfolio rule of all the agents different from $i$. From the strict convexity of the function $-\log (\cdot)$ and by the fact that agent $i$ has correct beliefs, one has

$$
\mu^{i}(w ; 1)>-\log \left(\delta+(1-\delta) \sum_{k=1}^{K}\left(\sum_{s=1}^{S} \pi_{s} d_{k, s}\right) \frac{x_{k}^{i^{c}}(w, x)}{x_{k}^{i}}\right)=0
$$

where in the latter we have used the definition of Subjective Generalized Kelly rule, R1. This immediately implies $\mu^{i}(1)>0$.

Now consider the case $v=\overline{0}$, one has

$$
\mu^{i}(w ; 0)=\sum_{s=1}^{S} \pi_{s} \log \left(\sum_{k=1}^{K} \frac{r_{k, s}(w ; x, \delta, D)}{x_{k}^{i^{c}}(w, x)} x_{k}^{i}\right)
$$

where $r_{k, s}(w ; x, \delta, D)$ is the $k, s$ element of the normalized payoff matrix $r(w ; x$, $\delta, D)$. To prove that $\mu^{i}(0)>0$, we show that $\mu^{i}(w ; 0)>0$ for any admissible $w$. First, we prove that $\lim _{\delta \rightarrow 0} \mu^{i}(w ; 0)>0$, and then we show that $\lim _{\delta \rightarrow 1} \mu^{i}(w ; 0)=0$.

For the first step one has

$$
\begin{aligned}
\frac{\partial \mu^{i}(w ; 0)}{\partial \delta}= & \sum_{s=1}^{S} \pi_{s}\left(\sum_{k=1}^{K} \frac{r_{k, s}(w ; x, \delta, D)}{x_{k}^{i^{c}}(w, x)} x_{k}^{i}\right)^{-1} \\
& \left(\sum_{k=1}^{K} \frac{x_{k}^{i}}{x_{k}^{i^{c}}(w, x)} \frac{\partial r_{k, s}(w ; x, \delta, D)}{\partial \delta}\right) .
\end{aligned}
$$

Its sign depends upon the derivative of the payoff matrix with respect to $\delta$. Using matrix differentiation, it reads

$$
\begin{aligned}
\frac{\partial r(w ; x, \delta, D)}{\partial \delta}= & -(\mathbb{I}-\delta A(w ; x))^{-1} D \\
& -(1-\delta)(\mathbb{I}-\delta A(w ; x))^{-1} A(w ; x)(\mathbb{I}-\delta A(w ; x))^{-1} D,
\end{aligned}
$$

and implies $\partial r_{k, s}(w ; x, \delta, D) / \partial \delta<0$ for any $k, s$.

For the second step, we use that $r_{k, s}(w ; x, \delta, D)=(1-\delta) d_{k, s}+\delta p_{k, s}(w ; x, \delta, D)$. Call $p$ the matrix whose $k, s$ element is $p_{k, s}(w ; x, \delta, D)$. Let us rewrite equation (10) in terms of normalized quantities, it reads

$$
(\mathbb{I}-\delta A(w ; x)) p=(1-\delta) A(w ; x) D .
$$

Thus in the limit of $\delta \rightarrow 1$ every column of $p$, call it $p_{s}$, solves the system

$$
(\mathbb{I}-A(w ; x)) p_{s}=\mathbf{0},
$$


which can be written as $A(w ; x) p_{s}=p_{s}$. Notice that, given $w$ with $w^{i}=0$, for each $s p_{s}=x^{i^{c}}(w, x)$ is a possible solution of the system. Moreover, since $\sum_{k=1}^{K}\left|A_{k, j}(w ; x)\right|=1 \forall j$ implies that $A(w, x)$ is a contraction, by Banach fixed point theorem such solution is unique. Thus,

$$
\lim _{\delta \rightarrow 1} \mu^{i}(w ; 0)=\sum_{s=1}^{S} \pi_{s} \log \left(\sum_{k=1}^{K} \frac{x_{k}^{i^{c}}(w, x)}{x_{k}^{i^{c}}(w, x)} x_{k}^{i}\right)=0
$$

Concluding we have shown that $\mu^{i}(1)>0$ and $\mu^{i}(0)>0$, hence by point (i) of Proposition 3 agent $i$ dominates and the Corollary is proven.

\section{A.8 Proof of Lemma 4}

From the definition of conditional drift

$$
\begin{aligned}
& \mu(0)=\sum_{s=1}^{S} \pi_{s} \log \left(\delta+(1-\delta) \sum_{k=1}^{K} d_{k, s} \frac{x_{k}^{1}}{x_{k}^{2}}\right) \text { and } \\
& \mu(1)=-\sum_{s=1}^{S} \pi_{s} \log \left(\delta+(1-\delta) \sum_{k=1}^{K} d_{k, s} \frac{x_{k}^{2}}{x_{k}^{1}}\right) .
\end{aligned}
$$

Considering that $0 \leq d_{k, s} \leq 1$ for all $s, k$ and that $\sum_{k=1}^{K} d_{k, s}=1$ for all $s$, we have

$$
\mu(0)>(1-\delta) \sum_{s=1}^{S} \pi_{s} \log \left(\sum_{k=1}^{K} d_{k, s} \frac{x_{k}^{1}}{x_{k}^{2}}\right) \geq(1-\delta) \sum_{k=1}^{K} x_{k}^{*} \log \left(\frac{x_{k}^{1}}{x_{k}^{2}}\right)
$$

and at the same time

$$
\mu(1)<-(1-\delta) \sum_{s=1}^{S} \pi_{s} \log \left(\sum_{k=1}^{K} d_{k, s} \frac{x_{k}^{2}}{x_{k}^{1}}\right) \leq(1-\delta) \sum_{k=1}^{K} x_{k}^{*} \log \left(\frac{x_{k}^{1}}{x_{k}^{2}}\right) .
$$

Putting together the two inequalities proves the assertion.

\section{A.9 Proof of Proposition 4}

The statement follows from the particular case of Proposition 3 when $N=2$ together with Lemma 4 and the definition of normalized prices. 


\section{A.10 Proof of Corollary 3}

The proof of the survival of agent 1 follows from Lemma 4 and Proposition 4. When agent 1 beliefs are correct, so that $x^{1}=x^{*}$, she also dominates since, exploiting the strict convexity of $-\log (\cdot)$,

$$
\begin{aligned}
\mu(1)= & \sum_{s} \pi_{s}\left(-\log \left(\delta+(1-\delta) \sum_{k=1}^{K} d_{k, s} \frac{x_{k}^{2}}{x_{k}^{*}}\right)\right)> \\
& -\log \left(\delta+(1-\delta) \sum_{k=1}^{K} \frac{x_{k}^{2}}{x_{k}^{*}} \sum_{s} \pi_{s} d_{k, s}\right) \\
= & -\log \left(\delta+(1-\delta) \sum_{k=1}^{K} \frac{x_{k}^{2}}{x_{k}^{*}} x_{k}^{*}\right)=0 .
\end{aligned}
$$

\section{A.11 Proof of Proposition 5}

The statement follows from the properties of the function $K L\left(x \| x^{*}\right): \Delta^{K} \rightarrow$ $\mathbb{R}_{+}, x \mapsto K L\left(x \| x^{*}\right)$. In particular, it is a continuous strictly convex function with a minimum equal to zero in $x=x^{*}$. Thus, it is defined over the compact set $\partial(\bar{\Delta})$ and there exists a minimum over this set because of the Weierstrass theorem. The strict convexity of $K L\left(x \| x^{*}\right)$ implies that it is also strictly quasi convex. This property together with the fact that $x^{*} \in \bar{\Delta}$ implies $\left\{x: K L\left(x \| x^{*}\right)<\mathcal{K}\right\} \subseteq \bar{\Delta}$. Hence, it is possible to choose a $\pi^{1} \neq \pi$ such that $K L\left(x^{1} \| x^{*}\right)=m<\mathcal{K}-\epsilon$ with $\epsilon>0$ and small enough. Then, one can easily define the set $\Pi=\left\{\pi^{\prime}: \pi^{\prime} \in \Delta_{+}^{S}, K L\left(x^{\prime} \| x^{*}\right)=m\right\}$ which has always at least two elements. Choosing $x^{1}$ and $x^{2}$ such that $\pi^{1}, \pi^{2} \in \Pi$ it is $\nabla_{x^{*}}\left(x^{2} \| x^{1}\right)=0$.

\section{A.12 Proof of Proposition 6}

An asset market economy with agents trading according to Subjective Generalized Kelly rules and an asset market economy with agents maximizing expected log-utilities under effective beliefs have, by construction, the same relative wealth dynamics. When markets are dynamically complete and agents maximize an expected log-utility, there is no loss of generality, assuming that they are trading all possible contingent commodities in date zero. In fact, all asset structures, as long as they are complete, allow agents to achieve the same consumption allocation, so that the relative wealth dynamics does not depend upon the asset structure. Under time-zero trading, it is well known that agents allocate in each state contingent good a fraction of wealth proportional to the state likelihood. In a two-agent economy, the relative wealth dynamics can thus be rewritten as

$$
\frac{w_{t+1}^{1}\left(\sigma_{t}, s_{t+1}\right)}{w_{t+1}^{2}\left(\sigma_{t}, s_{t+1}\right)}=\frac{\hat{\pi}_{s_{t+1}}^{1}\left(w_{t} ; \delta, D\right)}{\hat{\pi}_{s_{t+1}}^{2}\left(w_{t} ; \delta, D\right)} \frac{w_{t}^{1}\left(\sigma_{t}\right)}{w_{t}^{2}\left(\sigma_{t}\right)} \quad \forall \sigma_{t}, s_{t+1}, t .
$$


Applying the definition of the conditional drift $\mu(\cdot)$ to the log of the above process leads to the result.

\section{References}

Alchian, A.: Uncertainty, evolution, and economic theory. J. Polit. Econ. 58, 211-221 (1950)

Beker, P.F., Chattopadhyay, S.: Consumption dynamics in general equilibrium: a characterisation when markets are incomplete. J. Econ. Theory 145, 2133-2185 (2010)

Beker, P.F., Espino, E.: The dynamics of efficient asset trading with heterogeneous beliefs. J. Econ. Theory 146, 189-229 (2011)

Bektur, Ç.: Performance of investment strategies in the absence of correct beliefs. Decis. Econ. Finance 36(1), 23-37 (2013)

Bhamra, H., Uppal, R.: Asset prices with heterogeneity in preferences and beliefs. Rev. Financ. Stud. 27, 519-580 (2014)

Blume, L., Easley, D.: Evolution and market behavior. J. Econ. Theory 58, 9-40 (1992)

Blume, L., Easley, D.: If you are so smart why aren't you rich? Belief selection in complete and incomplete markets. Econometrica 74, 929-966 (2006)

Blume, L., Easley, D.: The market organism: Long-run survival in markets with heterogenous traders. J. Econ. Dyn. Control 33, 1023-1035 (2009)

Borovička, J.: Survival and long-run dynamics with heterogeneous beliefs under recursive preferences. Working paper, New York University (2015)

Bottazzi, G., Dindo, P.: Evolution and market behavior with endogenous investment rules. J. Econ. Dyn. Control 48, 121-146 (2014)

Bottazzi, G., Dindo, P.: Drift criteria for persistence of discrete stochastic processes on the line. LEM Working Paper 2015-26, Sant'Anna School for Advanced Studies, Pisa (2015)

Cao, D.: Speculation and financial wealth distribution under belief heterogeneity. Econ. J. (2017). doi:10. 1111/ecoj. 12519

Cogley, T., Sargent, T., Tsyrennikov, V.: Wealth dynamics in a bond economy with heterogeneous beliefs. Econ. J. 124, 1-30 (2013)

Condie, S.: Living with ambiguity: prices and survival when investors have heterogeneous preferences for ambiguity. Econ. Theory 36, 81-108 (2008). doi:10.1007/s00199-007-0264-1

Coury, T., Sciubba, E.: Belief heterogeneity and survival in incomplete markets. Econ. Theory 49, 37-58 (2012). doi:10.1007/s00199-010-0531-4

Cvitanić, J., Jouini, E., Malamud, S., Napp, C.: Financial markets equilibrium with heterogeneous agents. Rev. Finance 16(1), 285-321 (2012)

Cvitanić, J., Malamud, S.: Price impact and portfolio impact. J. Financ. Econ. 100(1), 201-225 (2011)

Dindo, P.: Survival in speculative markets. LEM Working Paper 2015-32, Scuola Superiore Sant'Anna, Pisa (2015)

Epstein, L., Zin, S.: Substitution, risk aversion, and the temporal behavior of consumption and asset returns: a theoretical framework. Econometrica 57, 937-969 (1989)

Evstigneev, I., Hens, T., Schenk-Hoppé, K.: Market selection of financial trading strategies: global stability. Math. Finance 12, 329-339 (2002)

Evstigneev, I., Hens, T., Schenk-Hoppé, K.: Evolutionary stable stock markets. Econ. Theory 27, 449-468 (2006)

Evstigneev, I., Hens, T., Schenk-Hoppé, K.: Globally evolutionary stable portfolio rules. J. Econ. Theory 140, 197-228 (2008)

Evstigneev, I., Hens, T., Schenk-Hoppé, K.: Evolutionary finance. In: Hens, T., Schenk-Hoppé, K. (eds.) Handbook of Financial Markets: Dynamics and Evolution. North-Holland (Handbooks in Economics Series) (2009)

Fedyk, Y., Heyerdahl-Larsen, C., Walden, J.: Market selection and welfare in a multi-asset economy. Rev. Finance 17(3), 1179-1237 (2013)

Friedman, M.: Essays in Positive Economics. Univ. Chicago Press, Chicago (1953)

Guerdjikova, A., Sciubba, E.: Survival with ambiguity. J. Econ. Theory 155, 50-94 (2015)

Hens, T., Schenk-Hoppé, K.: Handbook of Financial Markets: Dynamics and Evolution. North-Holland (Handbooks in Economics Series) (2009)

Horst, U., Wenzelburger, J.: On non-ergodic asset prices. Econ. Theory 34, 207-234 (2008) 
Jouini, E., Napp, C.: Heterogeneous beliefs and asset pricing in discrete time: an analysis of pessimism and doubt. J. Econ. Dyn. Control 30(7), 1233-1260 (2006)

Jouini, E., Napp, C.: Consensus consumer and intertemporal asset pricing with heterogeneous beliefs. Rev. Econ. Stud. 74(4), 1149-1174 (2007)

Jouini, E., Napp, C.: Unbiased disagreement in financial markets, waves of pessimism and the risk-return trade-off. Rev. Finance 15(3), 575-601 (2010)

Jouini, E., Napp, C.: Live fast, die young. Economic Theory 62, 265-278 (2016). doi:10.1007/ s00199-015-0894-7

Kelly, J.: A new interpretation of information rates. Bell Syst.Tech. J. 35, 917-926 (1956)

Lucas, R.: Asset prices in an exchange economy. Econometrica 46(6), 1429-1445 (1978)

Massari, F.: Markets with heterogeneous beliefs: a necessary and sufficient condition for a trader to vanish. J. Econ. Dyn. Control 78, 190-205 (2017)

Sandroni, A.: Do markets favor agents able to make accurate predictions. Econometrica 68(6), 1303-1341 (2000)

Sandroni, A.: Efficient markets and Bayes' rule. Econ. Theory 26, 741-764 (2005). doi:10.1007/ s00199-004-0567-4

Sciubba, E.: Asymmetric information and survival in financial markets. Econ. Theory 25, 353-379 (2005). doi:10.1007/s00199-003-0434-8

Sciubba, E.: The evolution of portfolio rules and the capital asset pricing model. Econ. Theory 29, 123-150 (2006). doi:10.1007/s00199-005-0013-2

Taussky, O.: A recurring theorem on determinants. Am. Math. Mon. 56, 672-676 (1949)

Yan, H.: Natural selection in financial markets: Does it work? Manag. Sci. 54, 1935-1950 (2008) 